\title{
Three-Component Vectorial Proper Orthogonal Decomposition of Axisymmetric Wake Behind a Disk
}

\author{
Murat Tutkun* \\ Chalmers University of Technology, 41296 Gothenburg, Sweden \\ Peter B. V. Johansson $\ddagger$ \\ Volvo Aero Corporation, 46161 Trolhättan, Sweden \\ and \\ William K. George \\ Chalmers University of Technology, 41296 Gothenburg, Sweden \\ DOI: $\underline{10.2514 / 1.31074}$
}

\begin{abstract}
One-component scalar, two-component vectorial, and three-component vectorial proper orthogonal decompositions of the axisymmetric turbulent wake have been studied to investigate possible effects of the number of components comprising the two-point correlation tensor forming the kernel of the proper orthogonal decompositions integral equation. A wind-tunnel experiment has been performed 50 diameters downstream of the wake generator, which was a disk of $20 \mathrm{~mm}$ in diameter. The Reynolds number based on the disk diameter was 20,400. Twelve cross hot wires were used to obtain the simultaneous multipoint measurements. Six of the probes were located on a fixed rake and the other six probes were located on a movable rake, which was traversed from 10 to $180 \mathrm{deg}$ in $10 \mathrm{deg}$ increments. Two experiments were carried out to obtain first the streamwise and azimuthal components of the velocity, and second to obtain the streamwise and radial components of the velocity. Seven out of nine components of the two-point correlation tensor were computed using measured velocities and the remaining two two-point correlations were extracted from the data using the continuity equations. The one-component scalar, and two- and three-component vectorial decomposition results were essentially in agreement. Eigenvalues for the full vector proper orthogonal decompositions integrated over frequency showed that Fourier mode 2 was the largest in both the streamwise and azimuthal velocity. Azimuthal mode 2 peaks at the near-zero frequency, whereas azimuthal mode 1 peaks at a frequency which corresponds to the vortex shedding frequency. Similar features in turbulence kinetic energy distributions were also observed in the modally decomposed two-point cross-spectra and two-point cross-correlations.
\end{abstract}

\section{Nomenclature}

$D=$ diameter of the disk, $\mathrm{mm}$

$d \quad=$ diameter of the suspension wires, $\mathrm{mm}$

$f \quad=$ frequency, $\mathrm{Hz}$

$l_{w} \quad=$ length of the sensing wire of hot-wire probe, $\mathrm{mm}$

$m=$ azimuthal mode number

$\mathrm{POD}_{u}=$ one-component scalar proper orthogonal decomposition of streamwise fluctuations

$\mathrm{POD}_{v}=$ one-component scalar proper orthogonal decomposition of radial fluctuations

$\mathrm{POD}_{w}=$ one-component scalar proper orthogonal decomposition of azimuthal fluctuations

$\mathrm{POD}_{u v}=$ two-component vectorial proper orthogonal decomposition of streamwise and radial fluctuations

$\mathrm{POD}_{u w}=$ two-component vectorial proper orthogonal decomposition of streamwise and azimuthal fluctuations

Presented as Paper 3300 at the 25th AIAA Aerodynamic Measurement Technology and Ground Testing Conference, San Francisco, CA, 5-8 June 2006; received 16 March 2007; revision received 15 November 2007; accepted for publication 20 January 2008. Copyright $\odot 2008$ by the American Institute of Aeronautics and Astronautics, Inc. All rights reserved. Copies of this paper may be made for personal or internal use, on condition that the copier pay the $\$ 10.00$ per-copy fee to the Copyright Clearance Center, Inc., 222 Rosewood Drive, Danvers, MA 01923; include the code 0001-1452/08 $\$ 10.00$ in correspondence with the CCC.

${ }^{*}$ Ph.D. Student, Department of Applied Mechanics; also at Norwegian Defence Research Establishment, Division of Protection, Post Office Box 25, NO-2027 Kjeller, Norway; murat.tutkun@chalmers.se.

${ }^{\dagger}$ Aerothermodynamics Engineer, Ph.D., Department of Aerothermodynamics; peter.pj.johansson@ volvo.com. AIAA Member.

Frofessor, Department of Applied Mechanics; wkgeorge@ chalmers.se. AIAA Associate Fellow.

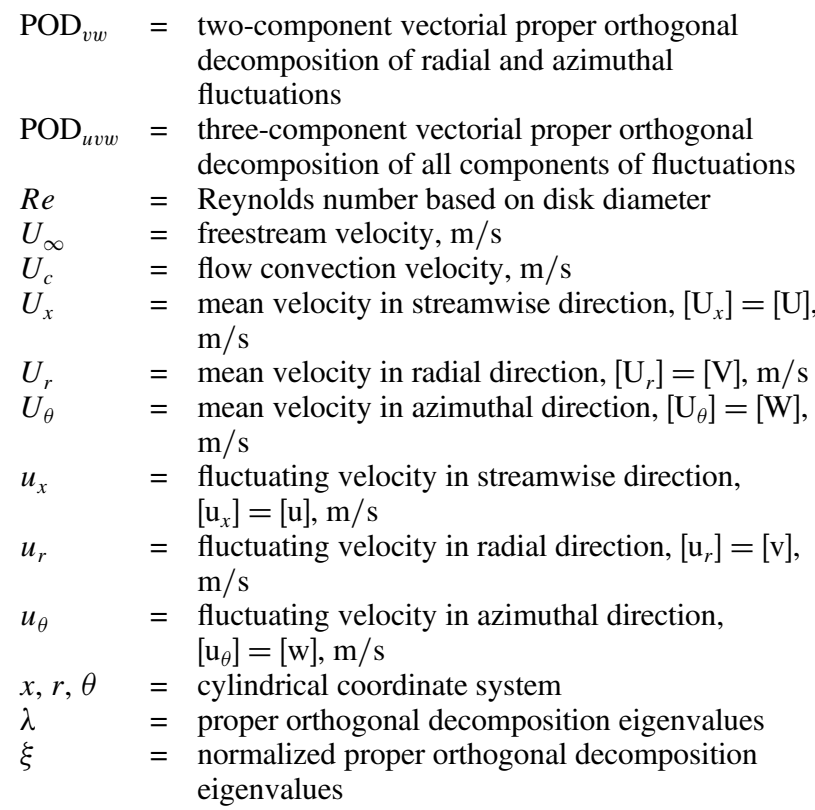

\section{Introduction}

$\mathbf{T}$ HE axisymmetric turbulent far wake was experimentally investigated using the proper orthogonal decomposition (POD) in this study. The main goal of this investigation was to address the effects of the number of velocity components involved in construction of the two-point cross-correlation tensor on the azimuthal distribution of the eigenvalues. For this purpose, 
comparison of the eigenvalue distribution and eigenspectra of the one-component scalar, two-component vectorial, and full, threecomponent vectorial decomposition of the velocity field were performed.

As a quantitative and unbiased method, Lumley [1] introduced the POD to identify and study the dynamics of the large-scale structures in turbulent fields with finite total energy. The POD provides an optimum deterministic description of the field, the so-called POD eigenvalues and eigenfunctions (or eigenmodes). These are obtained by seeking the largest projection onto the random velocity field in a mean square sense, maximization of which results in an integral eigenvalue problem (Fredholm integral equation of the second kind) in which the kernel is the two-point cross-correlation tensor of the velocity field. The POD has also been found very efficient at extracting the most energetic modes of the flow, and ordering them according to their energy content. Much information about the POD can be found in George [2] and Holmes et al. [3].

Even though the POD was introduced as an optimal and mathematical way of breaking the turbulence scales apart, utilization of the method took some time, mainly due to difficulties associated with the measurement and computation of the two-point crosscorrelation tensor. As pointed out by George [2], the POD needs sufficient information on the two-point cross-correlation tensor so that a complete space-time realization of the turbulent velocity field can be obtained. Computation of the cross-correlation tensor from the measured velocities is also difficult in terms of computing power and speed capabilities.

The first successful experimental implementations of the POD, almost two decades after Lumley's proposal [1] , came in the 1980s from Glauser [4] for high Reynolds number axisymmetric mixing layer and Herzog [5] for low Reynolds number pipe flow. Since then, experimental utilization of the POD has been widely used to break the turbulence scales apart [4,6-17]. Glauser [4], Glauser and George [6], and Glauser et al. [7] implemented the POD to investigate the coherent structures in an axisymmetric mixing layer. The axisymmetric shear layer was created by means of a round jet and the experiment was performed at 3 nozzle diameters downstream of the nozzle. It was shown that the orthogonal decomposition was very efficient at organizing the data such that the first POD mode contained $40 \%$ of the turbulence kinetic energy, whereas the second $40 \%$ of the energy was represented by the second and third POD modes. Following these studies, Glauser and George [18] performed an experiment using the same facility, but with a rake with cross-wire probes to examine differences between the one- and two-component decomposition, and found no significant effect due to including the radial component of the velocity in the analysis. The basic features of the extracted eigenspectra essentially were the same in that azimuthal mode 0 was dominant near the potential core and azimuthal modes 4 , 5 , and 6 were dominant outside of this region. This result motivated Citriniti [19], who used a rake of 138 single hot-wire probes and investigated the dynamics of the large-scale structures in a turbulent axisymmetric shear layer at the same downstream position. The results of this experiment supported the previous findings of Glauser and George [18]. An extension of this study for different downstream positions in both the near field [14] and far field [15] of an axisymmetric jet was performed to investigate the evolution of the most energetic modes. The near-field study for three different downstream locations, namely 2, 4, and 6 jet nozzle diameters downstream of the nozzle, showed that azimuthal mode 0 , which was the dominating mode of Citriniti and George [12], died off rapidly and the azimuthal mode 2 peaked shortly after the end of the potential core of the axisymmetric jet. The results of Gamard et al. [15] for the far-field region of the turbulent jet (15 to 69 jet nozzle diameters downstream of the nozzle) were also in agreement with these findings. They reported three major peaks in the azimuthal distribution of eigenvalues: one peak at azimuthal mode 0 at nearzero frequency, one peak at azimuthal mode 1 at a local Strouhal number of about one, and one small peak at the azimuthal mode 0 at near-zero frequency. They also showed that the eigenspectra for different downstream positions were essentially the same as the one obtained at the last downstream position of Jung et al. [14].
The experimental utilization of the POD has also been carried out for a number of other turbulent shear flows, including plane mixing layers and planar jets. Delville [8] used the POD technique to characterize the scales of the turbulent plane mixing layer by performing experiments similar to those of Glauser and George [18]. He carried out series of experiments [8] using one rake of single and cross hot-wire probes and compared one-component scalar and twocomponent vectorial decomposition. He reported an agreement between scalar and vectorial decomposition in terms of distribution of maximum energy, but noted that the vectorial POD represents the organizations in the flow more effectively because of the number of velocity components involved in the kernel of the problem. (Note that the nomenclature used by Delville [8] has been followed in the present study with an addition for three-component representation.) Further investigations on the same facility were carried out by Delville et al. [9] using two rakes of cross hot-wire probes. They applied two-component vectorial POD and then extended this work to three-component vectorial POD by using the spectral continuity equation in conjunction with the Taylor's frozen field hypothesis. Their results showed that the shapes of the eigenspectra were very similar for both cases. On the other hand, they also noted a small difference in the values of the low spanwise and streamwise wave number in the eigenspectra, and attributed this to the contribution of involvement of the third velocity component to the low wave numbers. Gordeyev and Thomas $[10,11]$ focused on the organized structures in the similarity region of the turbulent planar jet by applying the POD on single, twin, and triple rakes of cross hot-wire measurements. They also implemented the spectral continuity equation and analyzed the eigenvalue distribution based on the full three-dimensional kernel. The planar and nonplanar POD modes of the two- and three-component decompositions were found to be in agreement.

Recently, the axisymmetric wake behind a circular disk was investigated by performing multipoint single hot-wire measurements and using the POD $[13,16]$. These experiments started at $x / D=10$ and extended to $x / D=150$. It was observed that azimuthal Fourier mode 1 was dominant at the positions closest to the disk; however, this dominance got weaker as the flow evolved downstream and azimuthal Fourier mode 2 became the dominant mode. After $x / D \geq 40$, which can be considered as the start of the similarity region, azimuthal mode 2 was clearly the most energetic mode.

All of these experiments and POD analyses seemed to agree that the number of velocity components included in the two-point crosscorrelation tensor did not change the distribution of kinetic energy over the modes significantly. Therefore, it was of considerable surprise when Iqbal and Thomas [17] reported their results for the near-field region of an axisymmetric turbulent jet, between 3 to 12 nozzle diameters downstream the nozzle, using a three-component vectorial implementation of the POD by means of the spectral continuity equation. They reported that azimuthal mode 1 was the most energetic mode when the full Reynolds stress tensor was used, whereas azimuthal mode 2 was the largest when only the streamwise component was considered (consistent with the earlier results $[\underline{12}, \underline{14}, \underline{15}])$. Subsequently, Wänström et al. [20] carried out an axisymmetric jet experiment using stereoscopic particle image velocimetry (PIV) and investigated the azimuthal modal distribution of the turbulent kinetic energy via the snapshot POD. The results were in fact in agreement with what Iqbal and Thomas [17] observed, and the azimuthal mode 1 was found to be the dominant mode even in the far-jet region when the three-component vectorial POD was used.

This paper presents results of an investigation of the effects of the number of velocity components involved in the kernel of the POD integral equation for an axisymmetric far wake. The axisymmetric wake behind a disk was measured using a rake of cross hot-wire probes and the POD analysis of the data was performed. Following the previous applications of the spectral continuity equation $[9,11,17]$, a full, three-component implementation of the POD was carried out. The modally decomposed two-point cross-correlation and cross-spectra are discussed, and the eigenvalue distributions of 
the one-component scalar, two-component vectorial, and full, threecomponent vectorial POD are compared.

\section{Experimental Setup}

\section{A. Wind Tunnel and Suspension of the Disk}

The Chalmers L2 wind tunnel is a closed circuit, low-turbulence intensity wind tunnel with $60 \mathrm{~ms}^{-1}$ maximum speed. The freestream turbulence intensity of the tunnel is about $0.03 \%$. The test section cross section area is $1.80 \times 1.25 \mathrm{~m}^{2}$ and the length of the test section is $3 \mathrm{~m}$. The test section is octagonal because of corner fillets. The corner fillets decrease downstream to increase the cross section area to compensate for the boundary-layer growth. In the end of the test section, there is a 20 -mm-wide slot to match the pressure inside and outside of the test section. The slot also sucks the boundary layers which develop on the walls of the test section. The contraction ratio of the tunnel is 4.8. The tunnel has a flow temperature control unit which keeps the temperature constant with an accuracy of $\pm 0.1^{\circ} \mathrm{C}$.

The tunnel velocity during the experiment was monitored using a pitot tube connected to a Furness FCO510 digital micromanometer. The micromanometer was equipped with an absolute pressure sensor and a temperature sensor to provide the value of the tunnel velocity to the computer. The tunnel velocity was kept constant at $15.3 \mathrm{~ms}^{-1}$ and the corresponding Reynolds number based on disk diameter and the freestream velocity was 20,400. The measurement was performed at $1 \mathrm{~m}$ downstream of the disk, which corresponded to $x / D=50$. The momentum thickness $\theta$ and transverse length scale $\delta_{*}$ at this downstream location were found to be 7.1 and $42 \mathrm{~mm}$, respectively, which are in agreement with Johansson et al. [21], and Johansson and George [22].

A circular disk with a diameter of $20 \mathrm{~mm}$ was placed in the entrance of the test section using three pairs of suspension wires. The disk had a thickness of $2 \mathrm{~mm}$ with sharp edges and was made of aluminum. Steel piano wires were used to suspend the disk in the tunnel. Each suspension wire had a diameter of $0.2 \mathrm{~mm}$. Figure 1a shows the disk with suspension wires and the rake of cross hot-wire probes. As seen in the figure, the suspension wires were attached to the wind-tunnel wall asymmetrically so as not to impose a symmetry on the flow. The ratio between the area of the disk and the cross section area of the tunnel was less than $0.014 \%$, and so the flow blockage was clearly negligible.

The possible effect of suspension wires on the axisymmetric wake turbulence was recently investigated by Johansson and George [16], who performed the same axisymmetric wake experiment twice; first with three pairs of wires and then with four pairs of wires. They studied the differences in distribution of the turbulent kinetic energy and reported no detectable differences between the eigenvalue distributions of the two experiments. Therefore, the effects due to the suspension wires are assumed to be negligible in this study.

\section{B. Probe Configuration, Hot-Wire Anemometry, and Data Acquisition}

A detailed schematic of the rake with the numbering of the probes and the way of traversing the movable wing can be seen in Fig. 1b. Twelve cross hot-wire probes were used in a rake of two wings. Details about the mechanical properties of the rake can be found in Johansson and George [22]. Each wing carried six cross hot-wire probes radially spanning the wake. One of the wings was fixed as a reference point at zero degrees and the other was able to rotate around the centerline of the rake. The probes were equally spaced on the wings at a distance $\Delta r$ of $14 \mathrm{~mm}$, with the first probes located at $28 \mathrm{~mm}, 2 \Delta r$, away from the centerline.

The rake was originally designed for a 15 probe configuration and a minimum of $15 \mathrm{deg}$ angular separation between the wings $[16,22]$. To be able to decrease the minimum possible angular separation, the probe closest to the centerline from each wing was removed in this study. This allowed angular separation from 10 to $350 \mathrm{deg}$ in $10 \mathrm{deg}$ increments with an accuracy of $\pm 1 \mathrm{deg}$. The angular motion of the wing was performed by a completely automated, computer controlled traverse mechanism. No probe was located on the centerline of the rake in this study.

Brass telescopic tubes with an outer diameter of $4 \mathrm{~mm}$ were used as probe holders. One end of the probe holder was fixed to the rake and the other end held the probes. The lengths of the probe holders were $90 \mathrm{~mm}$ for the probes carried by the movable wing and $135 \mathrm{~mm}$ for those attached to the fixed wing. A ceramic tube (part no. R43, Scientific Instrument Services, Inc., Ringoes, New Jersey) with an outer diameter of $2.4 \mathrm{~mm}$ was used to insulate the prongs of the probes which were made of $0.4 \mathrm{~mm}$ steel piano wires. The ceramic tubing was placed in another brass tube with an outer diameter of $3.2 \mathrm{~mm}$ to assemble the probe. The distance between the probe holder and the end of the ceramic tube before the prongs was $60 \mathrm{~mm}$ for all probes. The lengths of the prongs after the ceramic tubing were 7.5 and $5.5 \mathrm{~mm}$ for the long ones and the short ones, respectively. The free ends of the prongs were cut by angle of $45 \mathrm{deg}$ to get the sensors normal to each other. The sensing wire was made of tungsten with a diameter of $5 \mu \mathrm{m}$ and a length of $2.8 \mathrm{~mm}$. The spacing between the sensors of the cross-wire probe was $0.7 \mathrm{~mm}$.

The wire length used in this study put some limitations on resolvable turbulence frequencies, because hot-wire probes only can resolve scales larger than twice the wire length. Therefore, the probes act as a low-pass spatial filter. On the other hand, this filtering property can be used to reduce spatial aliasing of turbulent velocity fluctuations as suggested by Glauser and George [23]. Further investigations on the spatial filtering due to finite size hot-wire sensors by Citriniti and George [24] demonstrated how the sensor filters out the turbulent kinetic energy at high frequencies. The cutoff frequency of the probe sensor was computed by [23]

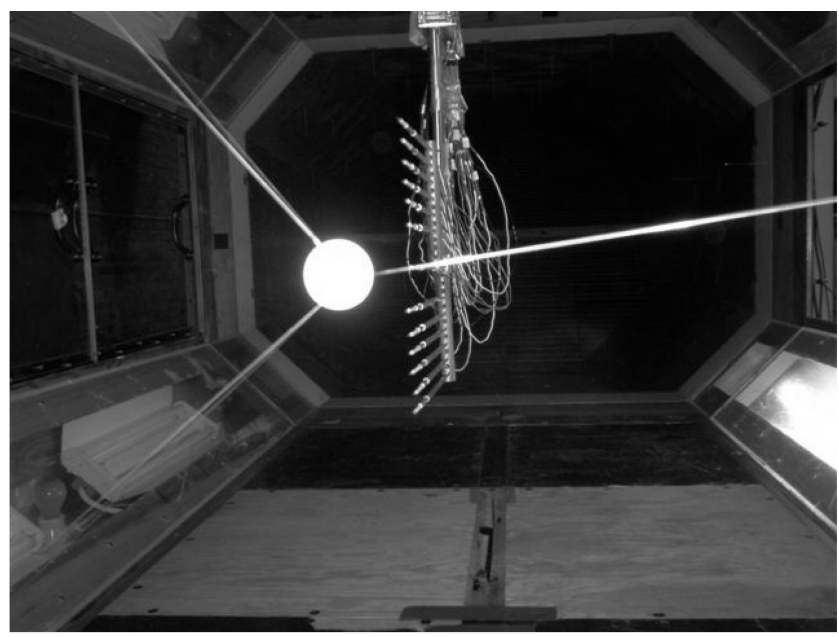

a) Tunnel and the rake

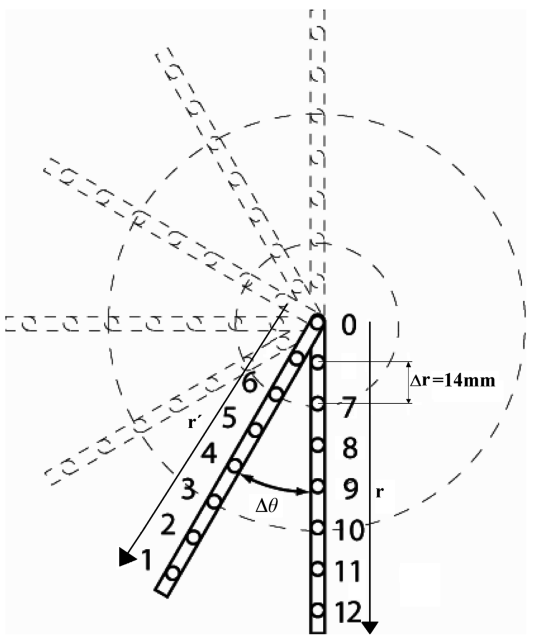

b) Traversing scheme

Fig. 1 Test section with the rake and the disk, and the traversing scheme. 


$$
f_{c}=\frac{U_{c}}{2 \times \ell_{w}}
$$

where $\ell_{w}$ was the probe sensor length and $U_{c}$ was the flow convection velocity. In this application, the freestream velocity of the tunnel $\left(15.3 \mathrm{~ms}^{-1}\right)$ was taken as the convective flow velocity due to the small velocity deficit of the far wake (approximately $2 \%$ of the freestream velocity at the corresponding downstream location). Therefore, the resulting wire cutoff frequency was found to be about $2730 \mathrm{~Hz}$, meaning that frequencies higher than this cutoff frequency were filtered out by the probe itself. This was not of concern in this investigation, because the primary purpose of this experiment was to study large, energetic scales and to apply the POD to determine the evolution of the energy containing turbulent structures (and not to resolve the small-scale turbulence).

Twenty-four single channel, Dantec Miniature-CTA 54T30 hotwire anemometers were used for the measurement. The analog outputs of the anemometers were digitized using an IO Tech Wavebook 51616 bit $1 \mathrm{MHz}$ sample and hold A/D converter with two expansion modules to enable 24 channel simultaneous sample and hold. The A/D converter was connected to the computer via a fast parallel port peripheral component interface (PCI) board. The control of sampling the data together with the traverse system was managed by a LabView program. The sampling frequency was set at $8 \mathrm{kHz}$ to satisfy the Nyquist criteria. The analog low-pass filters built into the Miniature-CTA were adjusted to $3 \mathrm{kHz}$ to avoid aliasing due to highfrequency electronic noise.

Measurements were made simultaneously for all 12 cross-wire probes through 24 channels. Sufficient information on the two-point cross-spectral tensor to apply the POD and obtain good convergence of the eigenfunctions was ensured by collecting 200 blocks of data per wire for each angular separation, which gave $7 \%$ variability for the power spectra. Each block had 8192 samples (corresponding to 2000 integral time scales), so that each block length was long enough to neglect the effect on the spectral measurement of the finite size record length $[23,25]$. There was a separation of 2000 integral time scales between each successive block to insure that each block was statistically independent.

Two sets of experiments were performed to obtain first the streamwise and azimuthal components of the velocity $\left(u_{x}\right.$ and $u_{\theta}$, respectively), and second the streamwise and radial components of the velocity ( $u_{x}$ and $u_{r}$, respectively). The second measurement was made possible by turning all the probes by 90 deg on their axes. The traversing of the rake from $10 \mathrm{deg}$ angular separation to $180 \mathrm{deg}$ separation between the wings was repeated by $10 \mathrm{deg}$ increments for both experiments.

\section{Effect of the Measurement Grid in $\Delta \theta$ and $r$}

The two-point correlations are homogeneous in the azimuthal direction and periodic over one revolution in this direction. Therefore, a Fourier series expansion in the azimuthal coordinate was optimal, at least in terms of representing the energy [2]. Fourier modes were used in this direction. A major concern of Fourier analysis in this type of experiment is that the turbulent kinetic energy at higher azimuthal Fourier modes could alias into the lower azimuthal Fourier modes [23]. To overcome the problem of spatial aliasing, the number of measurement positions should be more than twice the number of azimuthal modes which must be resolved. For the axisymmetric turbulent wake, 12 measurement points in the azimuthal direction were estimated as being enough to prevent the aliasing problem in corresponding modal space $[13,16]$, and so the angular separation between the wings and the increments was set at $15 \mathrm{deg}$. In the present study, the angular step size was decreased to $10 \mathrm{deg}$, yielding 18 measurement locations in the azimuthal direction to provide better resolution.

The measurement grid in the radial direction is also important because it must satisfy the minimal resolution to apply the POD. The maximum number of the POD eigenfunctions which can be extracted from the decomposition is limited by the number of radial positions at which the measurements have been taken and the number of velocity components included in the Reynolds stress tensor [8]. The sampling theorem of Glauser and George [23] states that if $\bar{M}$ eigenfunctions are required to represent the turbulent field, then at least $M$ measurement locations are required. Lumley [26] showed that the minimum number of eigenfunctions $M$ required to represent the inhomogeneous field, which is the radial direction in this study, is proportional to the ratio of the lateral extent of the flow and the integral scale in that direction. Following these suggestions, Johansson and George [16] used a measurement grid of $7 \times 7$ probes for their setup. They further investigated the grid size dependence of the eigenvalue distributions at $x / D=50$, and found very small differences only in eigenvalue of azimuthal mode 0 . They also reported that the first six POD modes cover $98 \%$ of total turbulence kinetic energy.

As noted in the previous section, the two innermost probes were removed in this experiment to be able to decrease the minimum azimuthal separation between the wings. Therefore, the possible effects of probe removal on the energy content of the field were investigated by checking the total energy distribution over the radial span. It was found by comparison with the previous results of Johansson and George [16] that removal of the innermost probes of the rakes did not affect the eigenvalue distribution.

\section{Calibration of Cross Hot-Wire Probes}

The cross-wire probes were calibrated simultaneously in the wind tunnel. A special traverse system was designed to be able to perform both the calibration in a range of $\pm 40 \mathrm{deg}$ and the angular rotation of the wings from 10 to $350 \mathrm{deg}$. Johansson et al. [13] and Johansson and George [22] reported that the root mean square of streamwise fluctuations of the axisymmetric wake at $x / D=50$ is about $2 \%$ of the freestream velocity, so that the probability of a \pm 4.5 deg change in the direction of the velocity vector, for the wake at this downstream location with $15.3 \mathrm{~ms}^{-1}$ freestream velocity, was found to be less than $0.1 \%$. Therefore, the angular calibration of the rake was performed between \pm 12 deg in 4 deg increments. The reference angle was $0 \mathrm{deg}$ for the rake at which the fixed wing was located during the experiments. Because of the low turbulence intensity, the effect of crossflow on the cross wire was negligible $[27,28]$.

Twelve different tunnel speeds varying between 11 and $18 \mathrm{~ms}^{-1}$ were used for the calibration. This range of tunnel speeds provided \pm 10 standard deviations around the freestream velocity of the experiment. For each tunnel velocity, seven angular variations of the probes with respect to tunnel velocity vector were scanned.

Effective cooling velocities for the cross-wire arrangement were written as formulated by Champagne et al. [29]. A linearizing scheme expressing the effective cooling velocities as the sum of powers of the anemometer output voltages was implemented [30]. A fourth-order polynomial was used for the linearization, even though orders lower than four could have been applied due to the low level of turbulence intensity in the far wake. This resulted in two nonlinear equations for each probe. In each nonlinear equation, output voltage, tunnel speed, and the angle between the flow axis and the probe axis were known. The unknowns of each equation (coefficients of the polynomial part of the equation, the angles between the normals of the wires, and the yaw coefficient) were determined using a nonlinear least-square curve fit. A built-in MATLAB function was used for this purpose.

This methodology yielded a functional relation between the effective cooling velocity and the anemometer output voltage for each wire. Once the voltage was read from the experiment, the effective cooling velocity for each wire was computed using the obtained functional relations. The ratio of the effective cooling velocities was rearranged using trigonometrical relations, and the resulting quadratic equation was solved for the angle between the normal of one of the wires and the flow velocity vector. By knowing this angle, it was straightforward to calculate the magnitude of velocity and the angle between the velocity vector and the probe axis. Finally, the velocity was decomposed into its components. 


\section{Background Theory}

\section{A. Formulation of the POD for an Axisymmetric Wake}

The POD seeks a deterministic field which has the maximum mean square projection onto the original random velocity field [1]. The maximization of the normalized mean square projection $\lambda$ results in a Fredholm integral equation of the second kind, where the symmetric kernel is the two-point cross-correlation tensor, which can be written for an axisymmetric flow as follows:

$$
\tilde{R}_{i, j}\left(x, x^{\prime} ; r, r^{\prime} ; \theta, \theta^{\prime} ; t, t^{\prime}\right)=\left\langle u_{i}(x, r, \theta, t) u_{j}\left(x^{\prime}, r^{\prime}, \theta^{\prime}, t^{\prime}\right)\right\rangle
$$

where' denotes different positions for $x, r$, and $\theta$, and a different time for $t$. Ensemble averaging is represented by \langle\rangle . The subscripts $i$ and $j$ denote appropriate components of the velocity, such as the streamwise velocity $u$ by $u_{x}$, the radial velocity $v$ by $u_{r}$, and the azimuthal velocity $w$ by $u_{\theta}$. Because the axisymmetric wake is stationary in time and statistically homogeneous in the azimuthal direction, the two-point cross-correlation tensor is a function of only the separations in these direction. If $\tau=t^{\prime}-t$ and $\Delta \theta=\theta^{\prime}-\theta$ represent the separations in time and in the azimuthal direction, respectively, then the two-point cross-correlation becomes

$$
\tilde{R}_{i, j}\left(x, x^{\prime} ; r, r^{\prime} ; \theta, \theta^{\prime} ; t, t^{\prime}\right)=R_{i, j}\left(x, x^{\prime} ; r, r^{\prime} ; \Delta \theta ; \tau\right)
$$

The proper orthogonal decomposition reduces to the Fourier decomposition in stationary and homogeneous directions $[\underline{1}, \underline{2}, \underline{26}]$. Also, if the flow is both homogeneous and periodic (as in the azimuthal direction), the decomposition in this direction becomes a Fourier series expansion. Therefore, Fourier transformations in time and the azimuthal direction are first performed on the two-point cross-correlation tensor, and then the proper orthogonal decomposition is applied to the Fourier coefficients, i.e., the cross-spectral tensor given by

$$
\begin{aligned}
& S_{i, j}\left(x, x^{\prime} ; r, r^{\prime} ; m ; f\right) \\
& \quad=\frac{1}{2 \pi} \int_{-\infty}^{\infty} \sum_{0}^{2 \pi} R_{i, j}\left(x, x^{\prime} ; r, r^{\prime} ; \Delta \theta ; \tau\right) e^{-i(2 \pi f \tau+i m \Delta \theta)} \mathrm{d} \tau \Delta \theta
\end{aligned}
$$

where $f$ is the frequency corresponding to $\tau$ and $m$ is the azimuthal Fourier mode number corresponding to $\Delta \theta$.

If only one downstream location is considered, as in this study, the $x$ in Eq. (4) is treated as a parameter and the POD integral equation reduces to the so-called slice POD $[\underline{6}, 10-14,16]$. On the other hand, because of the very low turbulence intensities of the threedimensional wake, to an excellent approximation the field can be considered frozen in time as it convects by the probes [31]. Thus, the streamwise dependent variable $x$ is identical to time, and the integral equation reduces simply to

$$
\int_{r^{\prime}} S_{i, j}\left(r, r^{\prime} ; m ; f\right) \phi_{j}^{*(n)}\left(r^{\prime} ; m ; f\right) r^{\prime} \mathrm{d} r^{\prime}=\lambda^{(n)}(m ; f) \phi_{i}^{(n)}(r ; m ; f)
$$

where $\lambda^{(n)}(m ; f)$ and $\phi_{i}^{(n)}(r ; m ; f)$ represent the eigenspectra and the eigenfunctions, respectively. Because domain is bounded in the inhomogeneous direction, the Hilbert-Schmidt theory applies.

The solution to this eigenvalue problem, Eq. (5), can be found using standard numerical schemes if the kernel is Hermitian symmetric. Because of the nature of the polar coordinate system of the axisymmetric wake, the $r^{\prime}$ on the left-hand side of Eq. (5) creates an asymmetry in the kernel. Hermitian symmetry of the kernel can be satisfied by multiplying both sides of the equation by $r^{1 / 2}$ and then rearranging the terms as follows:

$$
\begin{aligned}
\int_{r^{\prime}} \underbrace{r^{1 / 2} S_{i, j}\left(r, r^{\prime} ; m ; f\right) r^{\prime 1 / 2}}_{W_{i, j}\left(r, r^{\prime} ; m ; f\right)} \underbrace{\phi_{j}^{*(n)}\left(r^{\prime} ; m ; f\right) r^{\prime 1 / 2}}_{\hat{\phi}_{j}^{*(n)}\left(r^{\prime} ; m ; f\right)} \mathrm{d} r^{\prime} \\
=\underbrace{\lambda^{(n)}(m, f)}_{\hat{\lambda}^{(n)}(m ; f)} \underbrace{r^{1 / 2} \phi_{i}^{(n)}(r ; m ; f)}_{\hat{\phi}_{i}^{(n)}(r ; m ; f)}
\end{aligned}
$$

The rearranged POD integral equation with a Hermitian symmetric kernel can be written as follows:

$$
\int_{r^{\prime}} W_{i, j}\left(r, r^{\prime} ; m ; f\right) \hat{\phi}_{j}^{*(n)}\left(r^{\prime} ; m ; f\right) \mathrm{d} r^{\prime}=\hat{\lambda}^{(n)}(m ; f) \hat{\phi}_{i}^{(n)}(r ; m ; f)
$$

The number of the eigenvalues and eigenfunctions which the resulting eigenvalue equation [Eq. (7)] produces is determined by the velocity components included in the kernel. The number of eigenvalues can be easily computed by multiplying the number of grid points (hot-wire probe locations in this study) by the number of velocity components included in the computation of the two-point correlation tensor []].

\section{B. Spectral Analysis Technique and Numerical Implementation}

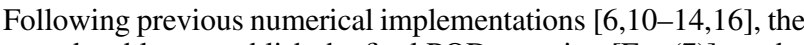
steps to be able to establish the final POD equation [Eq. (ㄱ) $]$ can be summarized as follows:

1) Instantaneous velocities are measured simultaneously at two points.

2) Fourier transformation of the instantaneous velocities is performed in time for the finite size record length, i.e.,

$$
\hat{u}_{i}(r, \theta, f)=\int_{-T / 2}^{T / 2} e^{-i 2 \pi f t} u_{i}(r, \theta, t) \mathrm{d} t
$$

where the $\hat{u}_{i}(r, \theta, f)$ are called the Fourier coefficients and $T$ represents the record length for each block of data. A fast Fourier transformation algorithm can be used to compute the Fourier coefficients efficiently.

3) Steps 1 and 2 are repeated for all pairs of points

4) Cross-spectra are computed and block averaging is performed as follows:

$$
\tilde{S}_{i, j}\left(r, r^{\prime} ; \Delta \theta ; f\right)=\frac{\left\langle\hat{u}_{i}(r, \theta, f) \hat{u}_{j}^{*}\left(r^{\prime}, \theta+\Delta \theta, f\right)\right\rangle}{T}
$$

where \langle\rangle and $*$ denote the block averaging and complex conjugate, respectively.

5) The doubly transformed cross-spectra are computed by the numerical approximation to the Fourier series expansion of Eq. (9) in the azimuthal direction, i.e.,

$$
S_{i, j}\left(r, r^{\prime} ; m ; f\right)=\frac{1}{2 \pi} \sum_{m=0}^{N} \tilde{S}_{i, j}\left(r, r^{\prime} ; \Delta \theta ; f\right) e^{-i m \Delta \theta} \mathrm{d}(\Delta \theta)
$$

where $N$ is twice the total number of angular separations $\Delta \theta$ in the azimuthal direction. Note that the second half of the measurement plane is created using the symmetry conditions as detailed here:

$$
\tilde{S}_{i, j}\left(r, r^{\prime} ; \Delta \theta ; f\right)=\tilde{S}_{j, i}^{*}\left(r^{\prime}, r ;-\Delta \theta ; f\right)
$$

where $i$ and $j$ denote appropriate components of the velocity as described for Eq. (2). We also performed measurements at $\Delta \theta=-80,-90$, and -100 to be able to check the accuracy of the symmetry conditions. The results were satisfactory and the difference in statistical quantities obtained at $\Delta \theta$ and $-\Delta \theta$ for these three angles was negligible.

6) The final eigenvalue problem is solved for each frequency and/ or azimuthal Fourier mode after construction of the Hermitian symmetric kernel. 


\section{Determination of Unmeasured Correlations}

Using cross-wire probes, only two components of the instantaneous velocity vector can be obtained simultaneously. In the polar coordinate system, these components will be either pairs of the streamwise and radial components of the velocity $\left(u_{x}, u_{r}\right)$ or pairs of the streamwise and azimuthal components of the velocity $\left(u_{x}, u_{\theta}\right)$. By two separate experiments to measure these two possible velocity combinations, seven members of the full two-point cross-correlation tensor can be directly computed using measured velocities. However, the cross-correlations between the radial and azimuthal velocities, $\left\langle u_{r}(x, r, \theta) u_{\theta}\left(x^{\prime}, r^{\prime}, \theta^{\prime}\right)\right\rangle$ and $\left\langle u_{\theta}(x, r, \theta) u_{r}\left(x^{\prime}, r^{\prime}, \theta^{\prime}\right)\right\rangle$, cannot be computed from the obtained velocities because the simultaneous measurement of pairs of the radial and azimuthal component are not possible with a single cross wire. On the other hand, the spectral continuity equation can be used to extract these unmeasured cross-correlations from the measured ones.

We start with the continuity equation at two points:

$$
\begin{gathered}
\frac{\partial u_{x}(x, r, \theta)}{\partial x}+\frac{1}{r} \frac{\partial\left[r u_{r}(x, r, \theta)\right]}{\partial r}+\frac{1}{r} \frac{\partial u_{\theta}(x, r, \theta)}{\partial \theta}=0 \\
\frac{\partial u_{x}\left(x^{\prime}, r^{\prime}, \theta^{\prime}\right)}{\partial x^{\prime}}+\frac{1}{r^{\prime}} \frac{\partial\left[r^{\prime} u_{r}\left(x^{\prime}, r^{\prime}, \theta^{\prime}\right)\right]}{\partial r^{\prime}}+\frac{1}{r^{\prime}} \frac{\partial u_{\theta}\left(x^{\prime}, r^{\prime}, \theta^{\prime}\right)}{\partial \theta^{\prime}}=0
\end{gathered}
$$

Multiplying Eq. (12) by $\mathrm{u}_{\theta}\left(x^{\prime}, r^{\prime}, \theta^{\prime}\right)$ and Eq. (13) by $\mathrm{u}_{\theta}(x, r, \theta)$, and taking the average of each equation, results in

$$
\begin{aligned}
& \frac{\partial R_{x, \theta}\left(r, r^{\prime}, \Delta \theta, \tau\right)}{\partial x}+\frac{1}{r} \frac{\partial\left[r R_{r, \theta}\left(r, r^{\prime}, \Delta \theta, \tau\right)\right]}{\partial r} \\
& +\frac{1}{r} \frac{\partial R_{\theta, \theta}\left(r, r^{\prime}, \Delta \theta, \tau\right)}{\partial \theta}=0 \\
& \frac{\partial R_{\theta, x}\left(r, r^{\prime}, \Delta \theta, \tau\right)}{\partial x^{\prime}}+\frac{1}{r^{\prime}} \frac{\partial\left[r^{\prime} R_{\theta, r}\left(r, r^{\prime}, \Delta \theta, \tau\right)\right]}{\partial r^{\prime}} \\
& +\frac{1}{r^{\prime}} \frac{\partial R_{\theta, \theta}\left(r, r^{\prime}, \Delta \theta, \tau\right)}{\partial \theta^{\prime}}=0
\end{aligned}
$$

Because of the low turbulence intensities in the far wake, Taylor's frozen field hypothesis can be used to replace the streamwise differentiation by the time differentiation such that $\partial / \partial x \approx$ $-\partial /\left(U_{c} \partial t\right)[31,32]$. Application of this hypothesis implies that Eqs. (14) and (15) can be rewritten as

$$
\begin{aligned}
&- \frac{1}{U_{c}} \frac{\partial R_{x, \theta}\left(r, r^{\prime}, \Delta \theta, \tau\right)}{\partial t^{\prime}}+\frac{1}{r} \frac{\partial\left[r R_{r, \theta}\left(r, r^{\prime}, \Delta \theta, \tau\right)\right]}{\partial r} \\
&+ \frac{1}{r} \frac{\partial R_{\theta, \theta}\left(r, r^{\prime}, \Delta \theta, \tau\right)}{\partial \theta}=0 \\
&- \frac{1}{U_{c}} \frac{\partial R_{\theta, x}\left(r, r^{\prime}, \Delta \theta, \tau\right)}{\partial t}+\frac{1}{r^{\prime}} \frac{\partial\left[r^{\prime} R_{\theta, r}\left(r, r^{\prime}, \Delta \theta, \tau\right)\right]}{\partial r^{\prime}} \\
&+\frac{1}{r^{\prime}} \frac{\partial R_{\theta, \theta}\left(r, r^{\prime}, \Delta \theta, \tau\right)}{\partial \theta^{\prime}}=0
\end{aligned}
$$

where $U_{c}$ represents the convection velocity, which is taken as the freestream velocity in this study due to the small velocity deficit $(<2 \%$ of the freestream velocity at $x / D=50)$. Note that the low turbulence intensity of the far wake (a few percent) makes this methodology much more reliable than its earlier applications to axisymmetric mixing layer [18], planar mixing layer [9], planar jet [11], and axisymmetric jet [17], where the turbulence intensity is substantially larger.

Because the field is stationary in time, differentiation with respect to $t$ and $t^{\prime}$ can be written in terms of $\tau=t^{\prime}-t$, i.e., $\partial / \partial t=-\partial / \partial \tau$ and $\partial / \partial t^{\prime}=\partial / \partial \tau$. Similarly, homogeneity in $\theta$ implies that differentiation with respect to $\theta$ can be converted to the differentiation with respect to $\Delta \theta=\theta^{\prime}-\theta$, i.e., $\partial / \partial \theta=-\partial / \partial(\Delta \theta)$ and $\partial / \partial \theta^{\prime}=\partial / \partial(\Delta \theta)$. Thus, Eqs. (16) and (17) can be reduced to

$$
\begin{aligned}
& \frac{1}{U_{c}} \frac{\partial R_{x, \theta}\left(r, r^{\prime}, \Delta \theta, \tau\right)}{\partial \tau}+\frac{1}{r} \frac{\partial\left[r R_{r, \theta}\left(r, r^{\prime}, \Delta \theta, \tau\right)\right]}{\partial r} \\
& -\frac{1}{r} \frac{\partial R_{\theta, \theta}\left(r, r^{\prime}, \Delta \theta, \tau\right)}{\partial(\Delta \theta)}=0 \\
& -\frac{1}{U_{c}} \frac{\partial R_{\theta, x}\left(r, r^{\prime}, \Delta \theta, \tau\right)}{\partial \tau}+\frac{1}{r^{\prime}} \frac{\partial\left[r^{\prime} R_{\theta, r}\left(r, r^{\prime}, \Delta \theta, \tau\right)\right]}{\partial r^{\prime}} \\
& \quad+\frac{1}{r^{\prime}} \frac{\partial R_{\theta, \theta}\left(r, r^{\prime}, \Delta \theta, \tau\right)}{\partial(\Delta \theta)}=0
\end{aligned}
$$

Finally, Fourier transformation of these equations with respect to $\tau$ and the Fourier series expansion in the azimuthal direction yields

$$
\begin{aligned}
& \frac{i 2 \pi f}{U_{c}} S_{x, \theta}\left(r, r^{\prime} ; m ; f\right)+\frac{1}{r} \frac{\partial\left[r S_{r, \theta}\left(r, r^{\prime} ; m ; f\right)\right]}{\partial r} \\
& -\frac{i m}{r} S_{\theta, \theta}\left(r, r^{\prime} ; m ; f\right)=0 \\
& -\frac{i 2 \pi f}{U_{c}} S_{\theta, x}\left(r, r^{\prime} ; m ; f\right)+\frac{1}{r^{\prime}} \frac{\partial\left[r^{\prime} S_{\theta, r}\left(r, r^{\prime} ; m ; f\right)\right]}{\partial r^{\prime}} \\
& +\frac{i m}{r^{\prime}} S_{\theta, \theta}\left(r, r^{\prime} ; m ; f\right)=0
\end{aligned}
$$

Rearranging the resulting equations and integrating from $r$ to $\infty$ yields equations for computing the unmeasured correlations in the frequency and Fourier modal space:

$$
\begin{aligned}
& S_{r, \theta}\left(r, r^{\prime} ; m ; f\right)=\frac{i 2 \pi f}{r U_{c}} \int_{r}^{\infty} r S_{x, \theta}\left(r, r^{\prime} ; m ; f\right) \mathrm{d} r \\
& \quad-\frac{i m}{r} \int_{r}^{\infty} S_{\theta, \theta}\left(r, r^{\prime} ; m ; f\right) \mathrm{d} r \\
& S_{\theta, r}\left(r, r^{\prime} ; m ; f\right)=-\frac{i 2 \pi f}{r^{\prime} U_{c}} \int_{r^{\prime}}^{\infty} r^{\prime} S_{\theta, x}\left(r, r^{\prime} ; m ; f\right) \mathrm{d} r^{\prime} \\
& \quad+\frac{i m}{r^{\prime}} \int_{r^{\prime}}^{\infty} S_{\theta, \theta}\left(r, r^{\prime} ; m ; f\right) \mathrm{d} r^{\prime}
\end{aligned}
$$

\section{Results}

\section{A. Two-Point Cross-Spectra}

The primary purpose of this study is to investigate possible effects of the number of velocity components used in the construction of the Reynolds stress tensor on the eigenvalue distribution of the proper orthogonal decomposition of the axisymmetric wake. The modally decomposed two-point cross-spectra of the streamwise-streamwise, radial-radial, and azimuthal-azimuthal components of the velocity fluctuations for $r=r^{\prime}$ are shown in Figs. 2-4, respectively. In these figures, only first the six azimuthal modes up to $750 \mathrm{~Hz}$ are shown, because there is no pronounced activity at higher azimuthal modes and frequencies. Only the real parts of the cross-spectra are shown because the magnitude of the imaginary part is significantly smaller than the real parts.

One of the distinct features seen in two-point cross-spectra of all fluctuating velocities is the azimuthal Fourier mode 1 peak at the shedding frequency of $97 \mathrm{~Hz}$. This is consistent with the observations of Johansson and George [16], and suggests that the initial condition effects associated with the vortex shedding are mainly carried by the azimuthal Fourier mode 1 . The peak at $97 \mathrm{~Hz}$ is observed over the entire radial span of the wake. In the case of the streamwisestreamwise two-point cross-spectra as seen in Fig. 2, there is almost no sign of the vortex shedding in the other azimuthal Fourier modes. The amount of turbulence kinetic energy of the coherent azimuthal structures represented by the azimuthal Fourier mode 1 is of the same order of magnitude as azimuthal Fourier modes 0 and 2 at the radial location closest to the centerline. This changes drastically at the 
following radial positions toward the edge of the wake, where most of the turbulence kinetic energy is instead carried by the azimuthal Fourier mode 2 . The azimuthal mode 1 peak at the vortex shedding frequency is always very sharp and followed by a sudden decrease in the energy level to almost zero. Most of the turbulence kinetic energy at the radial positions larger than $2 \Delta r$ is due to the coherent structures associated with the azimuthal Fourier mode 2. The main feature of the azimuthal Fourier mode 2 is the peak at near-zero frequency. This is observed for all radial positions. The largest amount of turbulence kinetic energy at the azimuthal mode 2 exists in the second radial position, $r=3 \Delta r$.

Figure 3 shows the two-point cross-spectra of the radial velocity fluctuations, which exhibit quite different behavior than those of the streamwise velocity fluctuations, especially for the azimuthal Fourier modes 0 and 1 . Azimuthal mode 0 does not have any energy at frequencies lower than the vortex shedding frequency. This is true for

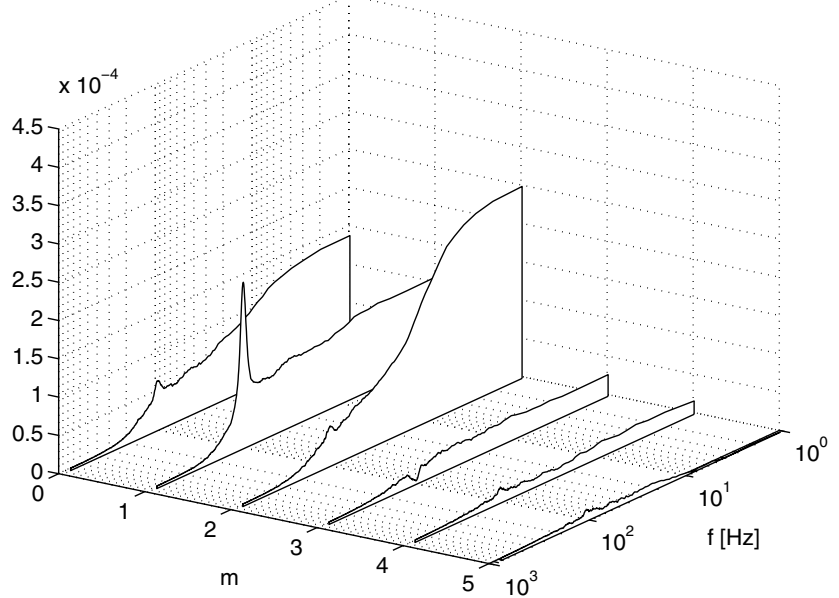

a)

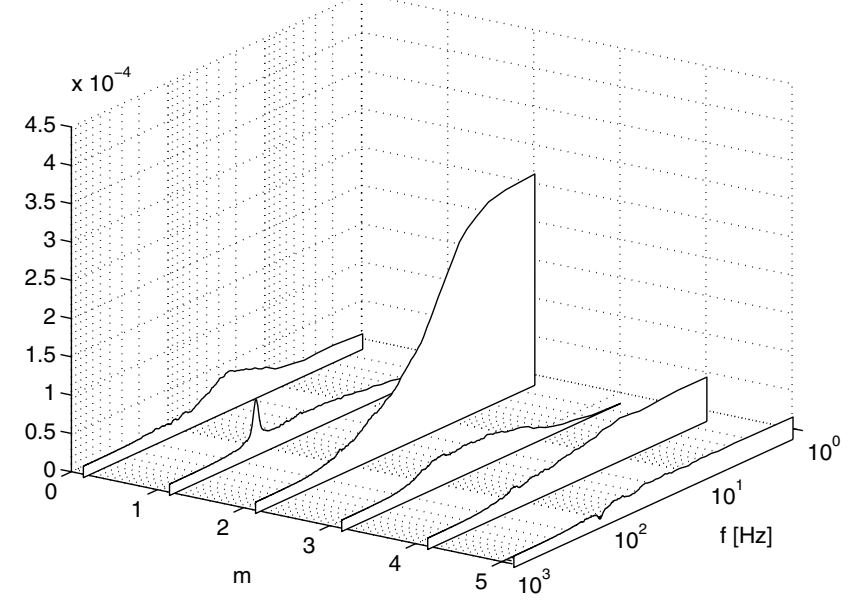

c)

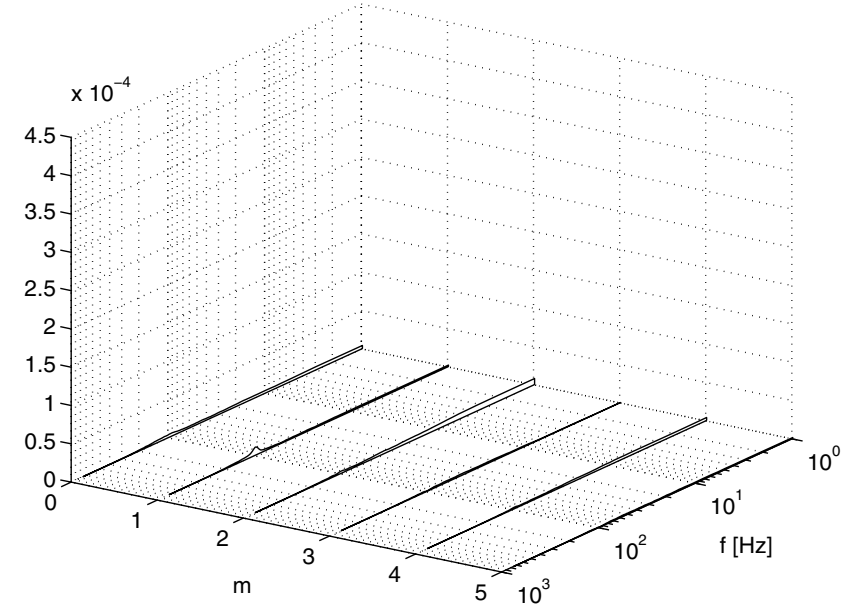

e)

Fig. 2 Two-point cross-spectrum $S_{x, x}\left(r, r^{\prime} ; m ; f\right)$ as function of azimuthal mode number $m$ and frequency $f$ at different radial positions: a) $r=r^{\prime}=2 \Delta r=0.67 \delta_{*}, \quad$ b) $r=r^{\prime}=3 \Delta r=1.00 \delta_{*}, \quad$ c) $r=r^{\prime}=4 \Delta r=1.33 \delta_{*}, \quad$ d) $\quad r=r^{\prime}=5 \Delta r=1.67 \delta_{*}, \quad$ e) $\quad r=r^{\prime}=6 \Delta r=2.00 \delta_{*}$, f) $r=r^{\prime}=7 \Delta r=2.33 \delta_{*}$.

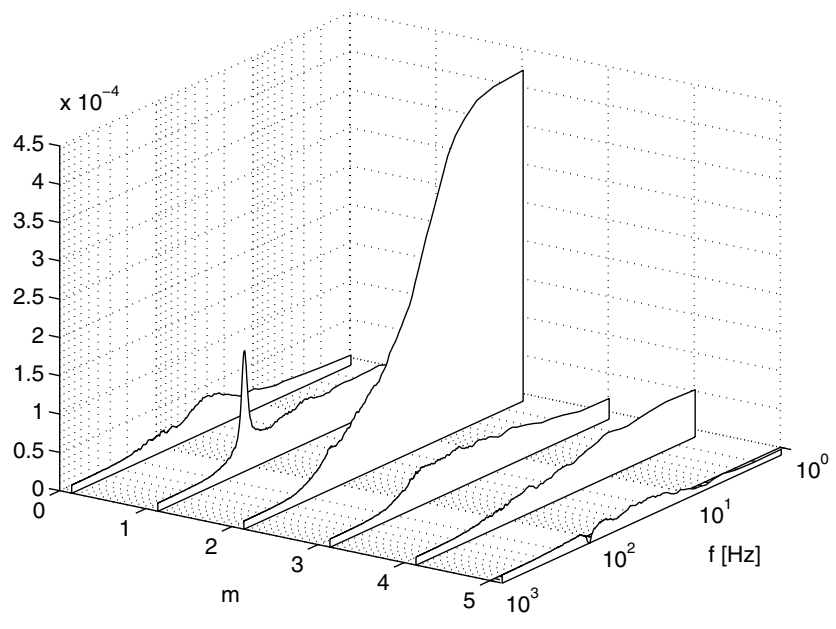

b)

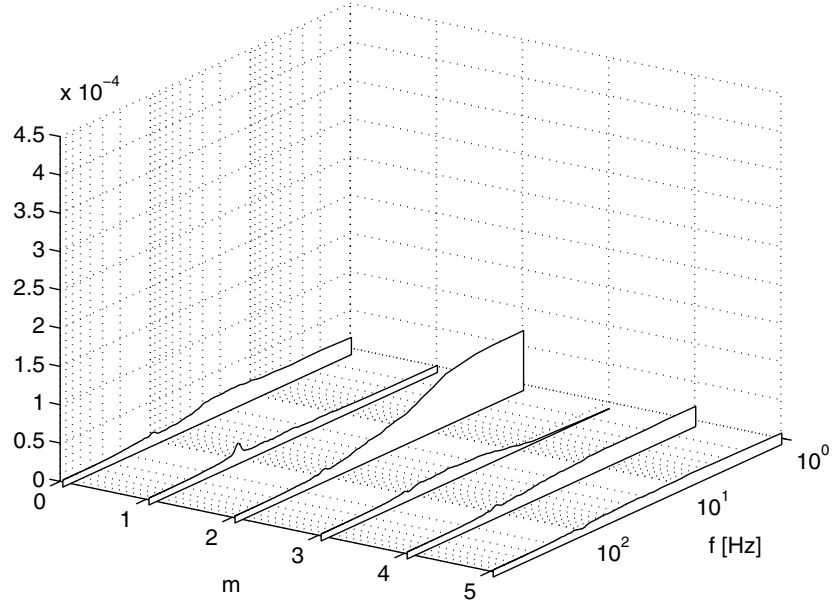

d)

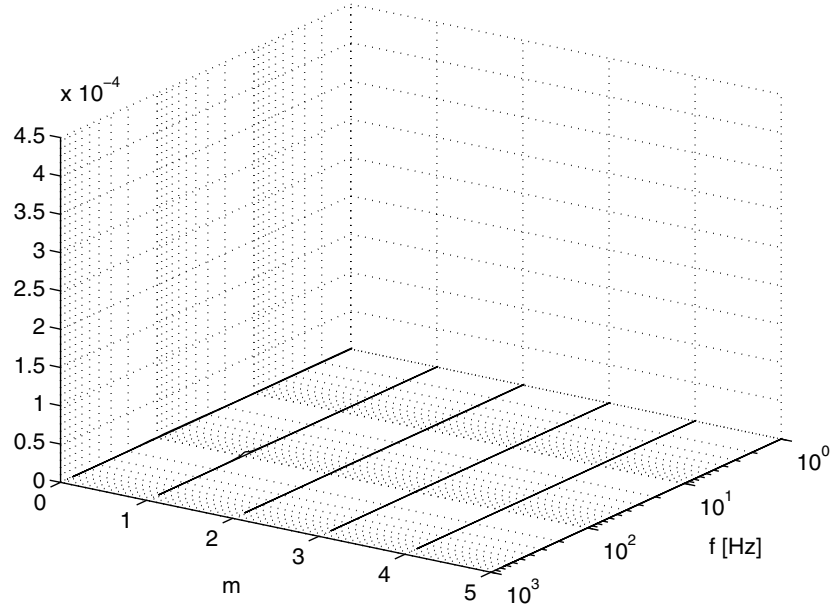

f) 
all radial positions across the span of the wake. The kinetic energy at the azimuthal mode 0 increases considerably at the shedding frequency and then rolls off slowly at higher frequencies. Azimuthal mode 0 peak has its maximum at the innermost probe location among different radial positions and decays in the radial direction.

There also exists an azimuthal Fourier mode 1 peak at the shedding frequency for the radial fluctuating velocities similar to the streamwise fluctuations, but it is more pronounced in this case, as

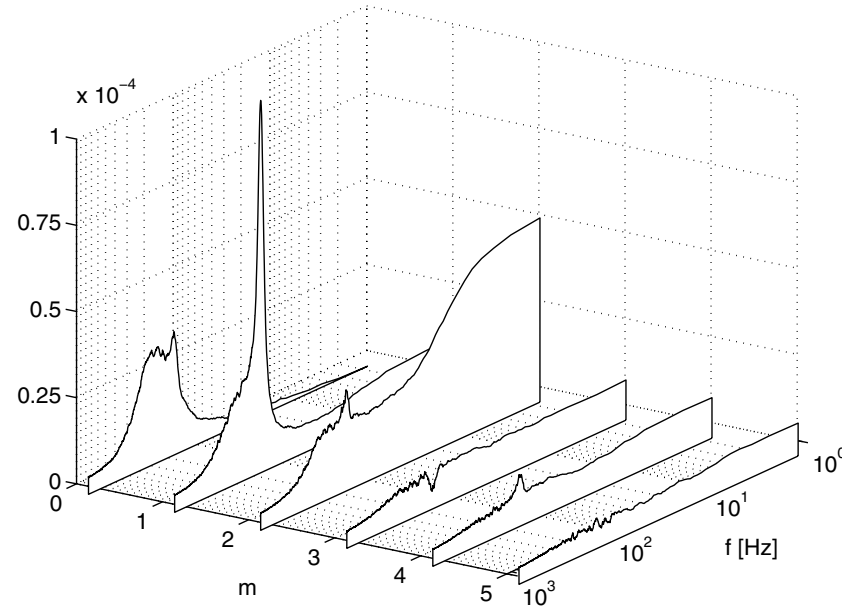

a)

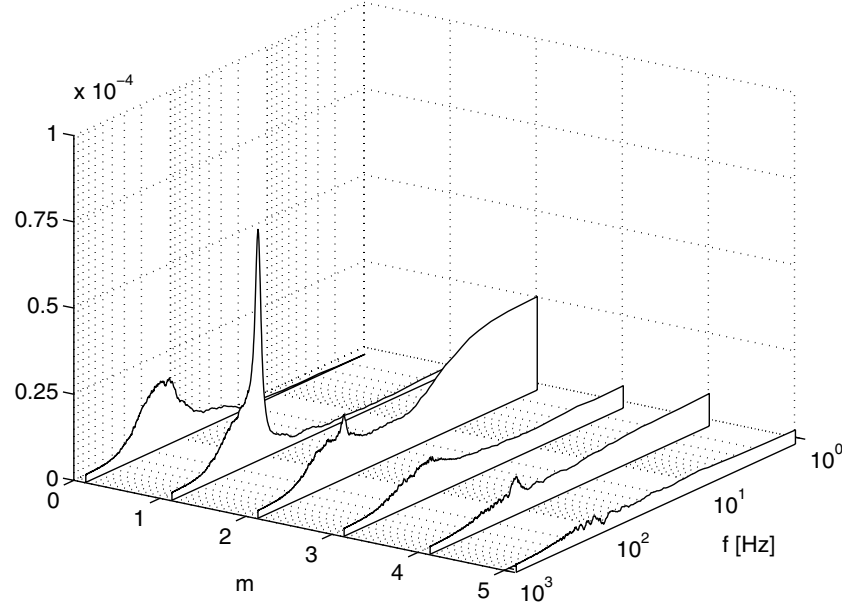

c)

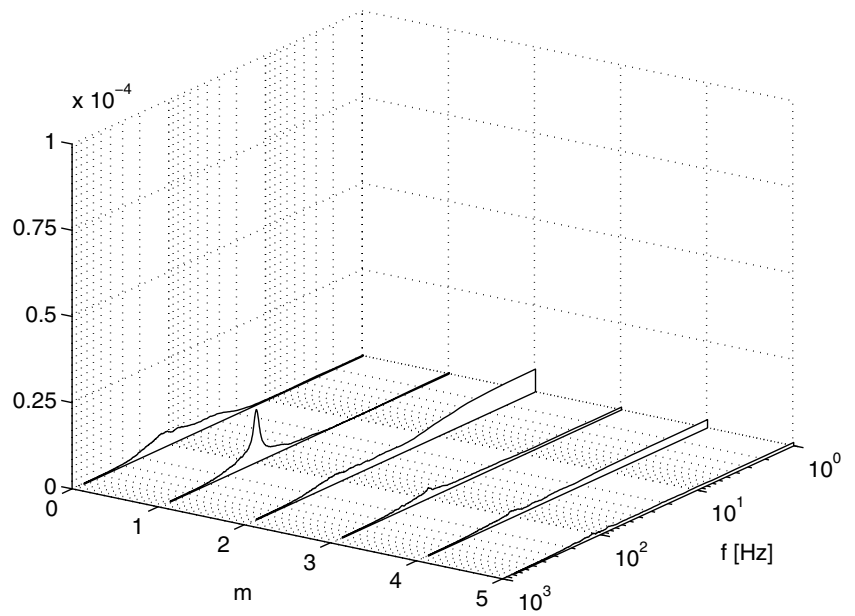

e)

Fig. 3 Two-point cross-spectrum $S_{r, r}\left(r, r^{\prime} ; m ; f\right)$ as function of azimuthal mode number $m$ and frequency $f$ at different radial positions: a) $r=r^{\prime}=2 \Delta r=0.67 \delta_{*}, \quad$ b) $r=r^{\prime}=3 \Delta r=1.00 \delta_{*}, \quad$ c) $r=r^{\prime}=4 \Delta r=1.33 \delta_{*}, \quad$ d) $\quad r=r^{\prime}=5 \Delta r=1.67 \delta_{*}, \quad$ e) $\quad r=r^{\prime}=6 \Delta r=2.00 \delta_{*}$, f) $r=r^{\prime}=7 \Delta r=2.33 \delta_{*}$. shown in Fig. 3. The turbulence kinetic energy of azimuthal mode 1 at all radial positions, except the innermost probe location, first appears at the shedding frequency for the radial fluctuations. Frequencies lower than the shedding frequency do not show any turbulence activity.

Azimuthal Fourier mode 2 in the two-point cross-spectra of the radial velocity fluctuations has its peak at near-zero frequency, similar to the cross-spectra of the streamwise fluctuations. However,

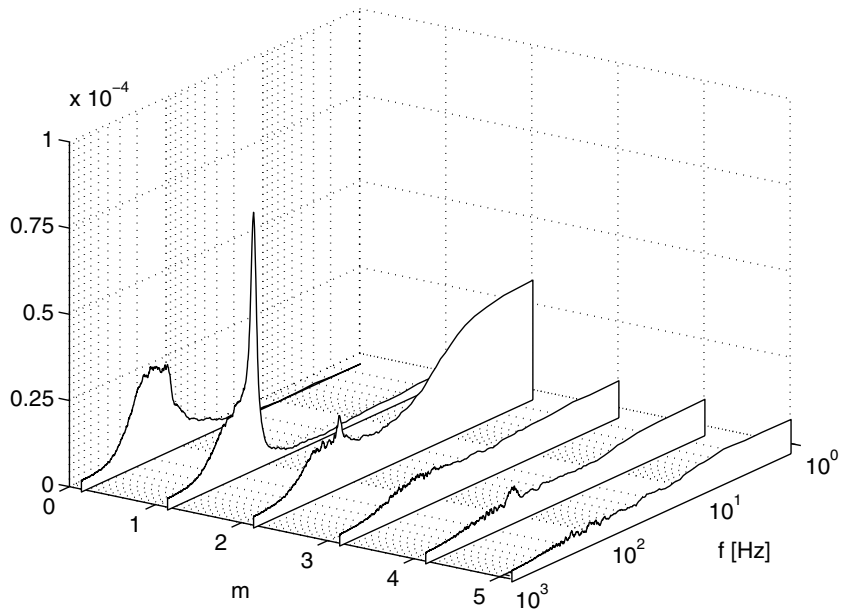

b)

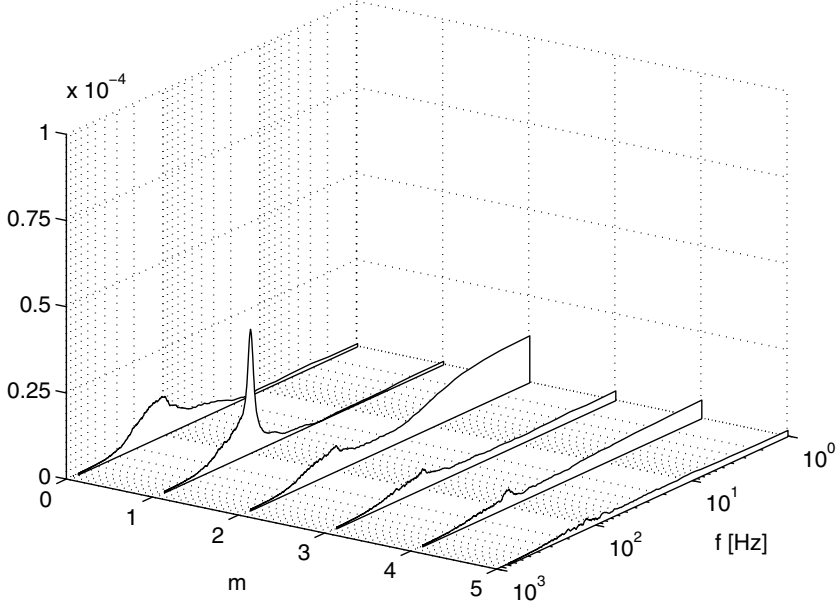

d)

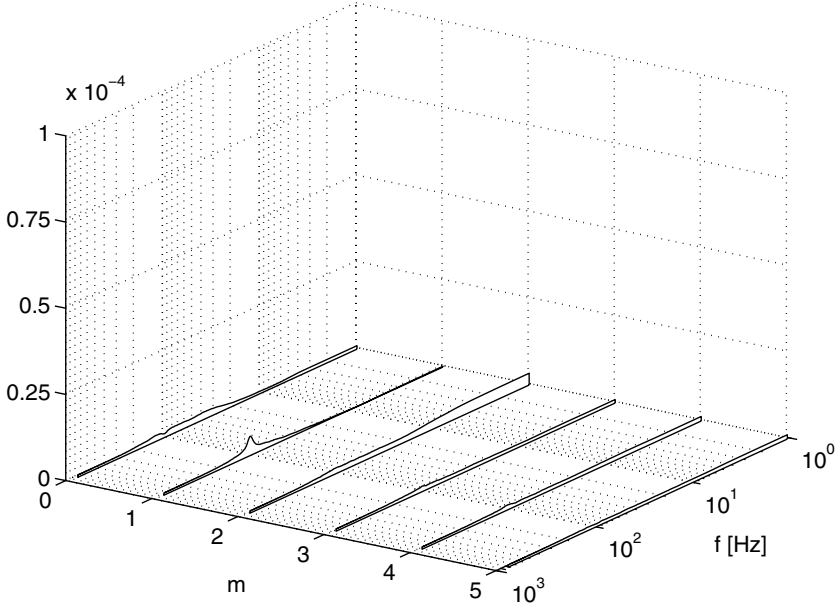

f) 
there are some important differences between Figs. $\underline{2}$ and $\underline{3}$ involving azimuthal mode 2, which can be listed as follows:

1) The effect of vortex shedding also appears at the shedding frequency of azimuthal mode 2 .

2) The amount of the turbulence kinetic energy of the azimuthal mode 2 is on the same order of magnitude, or less than, that of the azimuthal mode 1 .

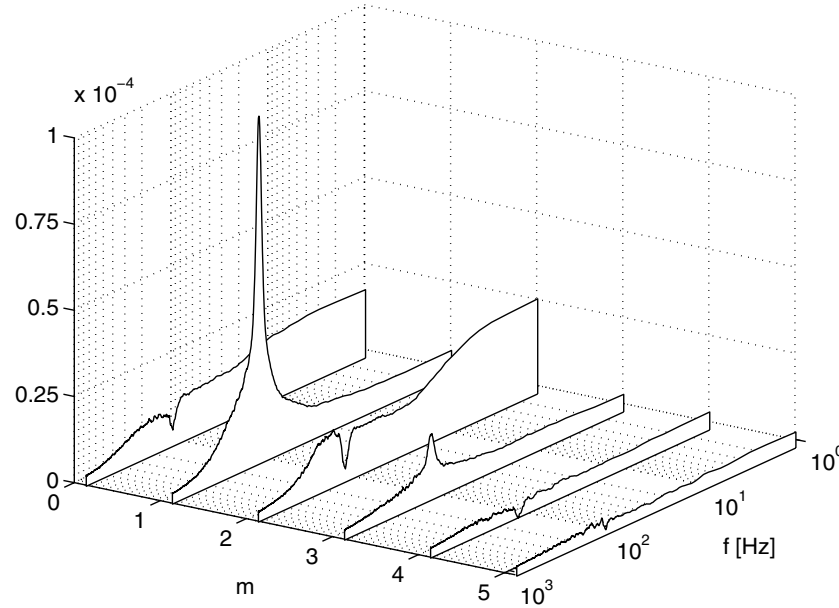

a)

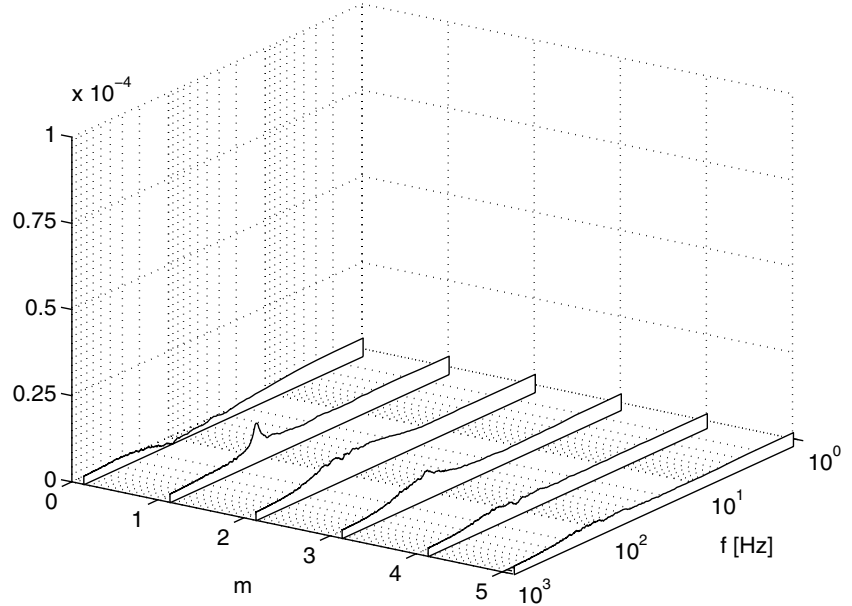

c)

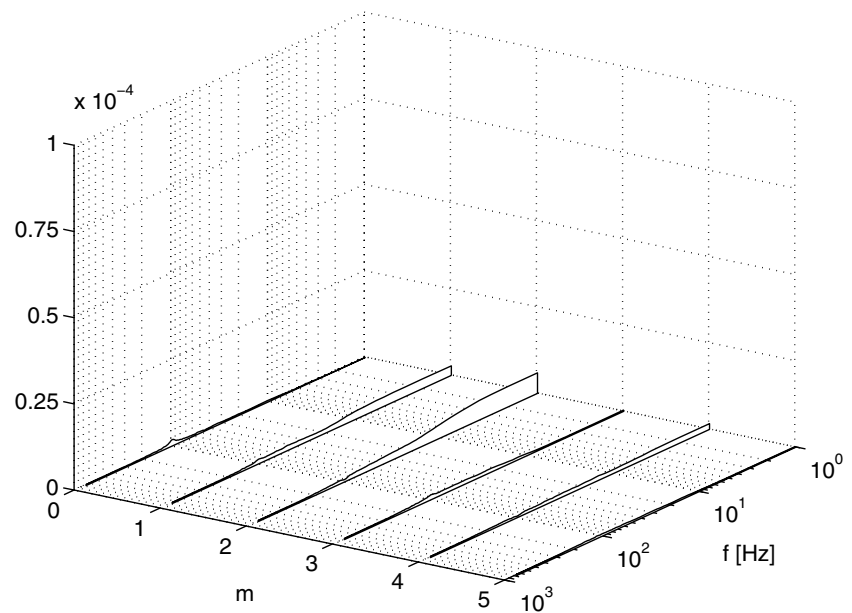

e)

Fig. 4 Two-point cross-spectrum $S_{\theta, \theta}\left(r, r^{\prime} ; m ; f\right)$ as function of azimuthal mode number $m$ and frequency $f$ at different radial positions: a) $r=r^{\prime}=2 \Delta r=0.67 \delta_{*}, \quad$ b) $r=r^{\prime}=3 \Delta r=1.00 \delta_{*}, \quad$ c) $r=r^{\prime}=4 \Delta r=1.33 \delta_{*}, \quad$ d) $\quad r=r^{\prime}=5 \Delta r=1.67 \delta_{*}, \quad$ e) $\quad r=r^{\prime}=6 \Delta r=2.00 \delta_{*}$, f) $r=r^{\prime}=7 \Delta r=2.33 \delta_{*}$.
3) The total turbulence kinetic energy of azimuthal mode 2 has its maximum at the innermost probe location and decreases with increasing $r$.

Two-point cross-spectra of the azimuthal velocity fluctuations are shown in Fig. 4. A very strong azimuthal mode 1 peak at the shedding frequency is observed at the first radial position. The strength of azimuthal mode 1 peak goes down for the second radial position and

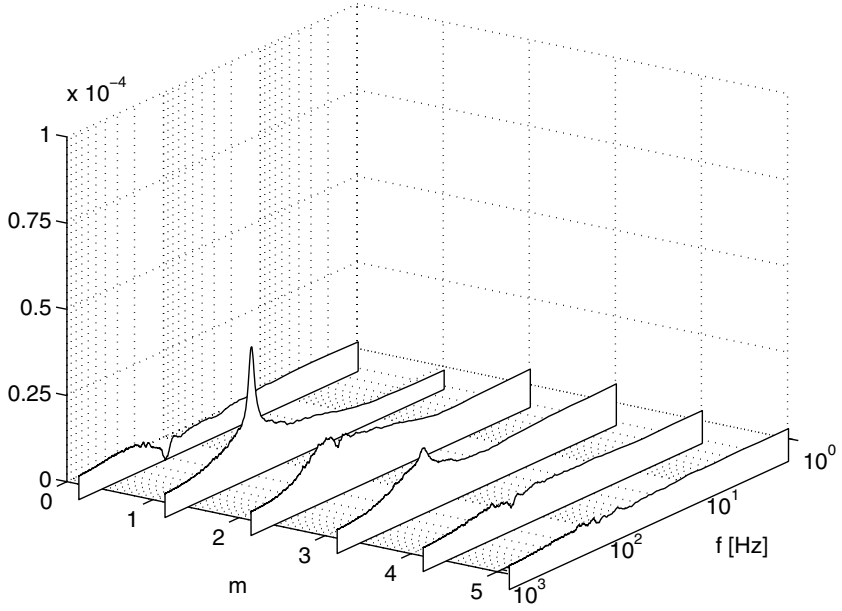

b)

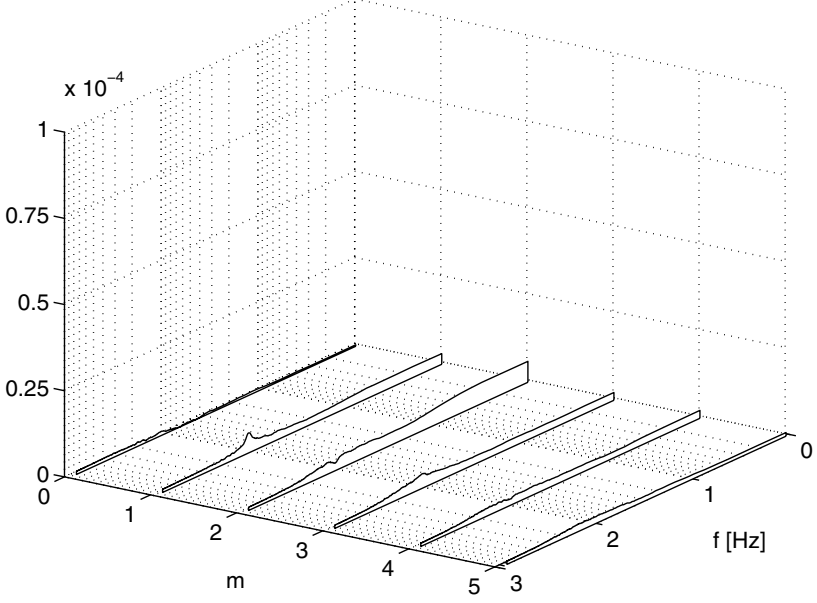

d)

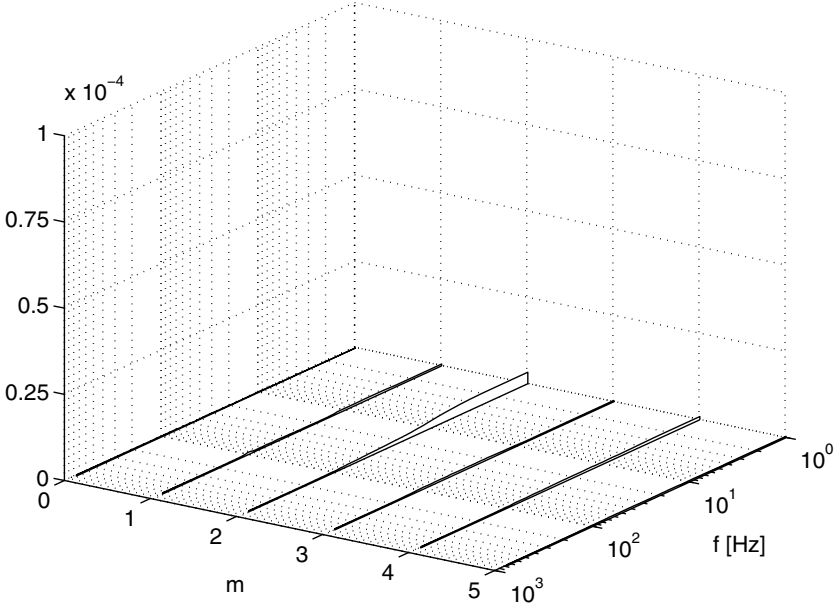

f) 
it continues to decay for the next radial position outward. The most interesting observation from the cross-spectra of the azimuthal fluctuations is that there is always a peak at the shedding frequency for odd azimuthal modes. On the contrary, there is a trough at the shedding frequency for even azimuthal modes. Azimuthal modes 0 and 2 have similar shapes for $r=2 \Delta r$; however, mode 0 dies off faster than mode 2 in the radial outward direction in the wake. For the azimuthal fluctuations, the turbulence energy captured by the azimuthal modes 1 and 2 are at the same order of magnitude as can be seen in Fig. 4.

The total turbulence kinetic energy distribution over different azimuthal modes and frequencies is presented in Fig. 5. The total was found by summing the kinetic energies from all three components of fluctuations. Therefore, $S_{k, k}\left(r, r^{\prime} ; m ; f\right)$ is simply equal to

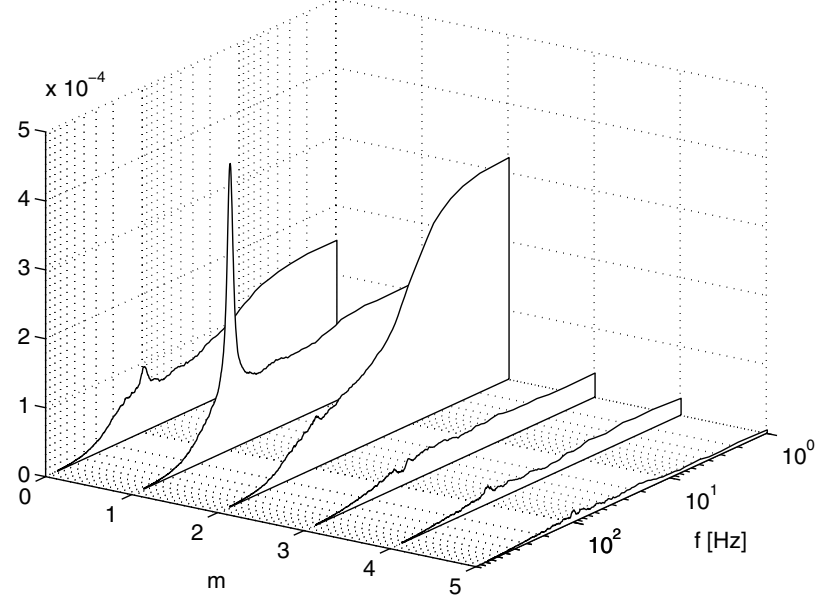

a)

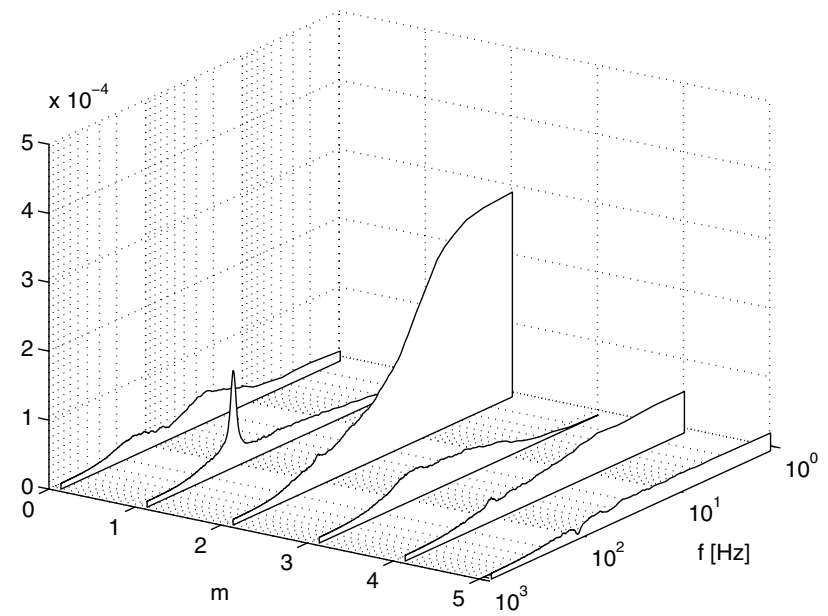

c)

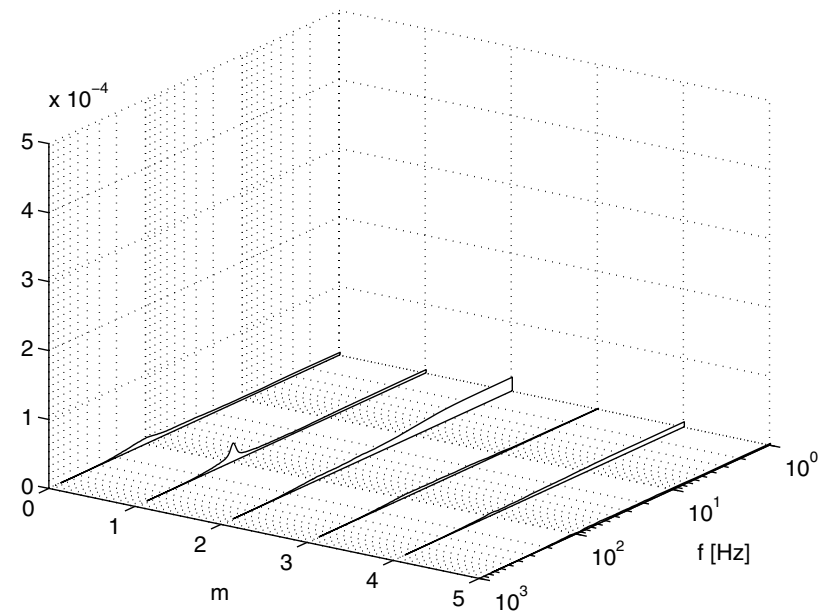

e)

Fig. 5 Two-point cross-spectrum $S_{k, k}\left(r, r^{\prime} ; m ; f\right)=S_{x, x}\left(r, r^{\prime} ; m ; f\right)+S_{r, r}\left(r, r^{\prime} ; m ; f\right)+S_{\theta, \theta}\left(r, r^{\prime} ; m ; f\right)$ as function of azimuthal mode number $m$ and frequency $f$ at different radial positions: a) $r=r^{\prime}=2 \Delta r=0.67 \delta_{*}$, b) $r=r^{\prime}=3 \Delta r=1.00 \delta_{*}$, c) $\left.r=r^{\prime}=4 \Delta r=1.33 \delta_{*}, d\right) r=r^{\prime}=5 \Delta r=1.67 \delta_{*}$, e) $r=r^{\prime}=6 \Delta r=2.00 \delta_{*}$, f) $r=r^{\prime}=7 \Delta r=2.33 \delta_{*}$.

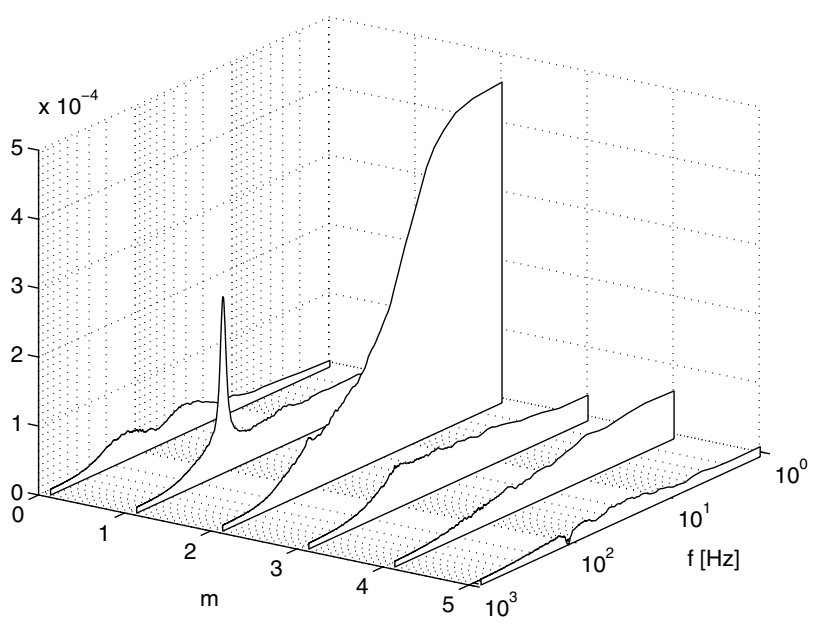

b)

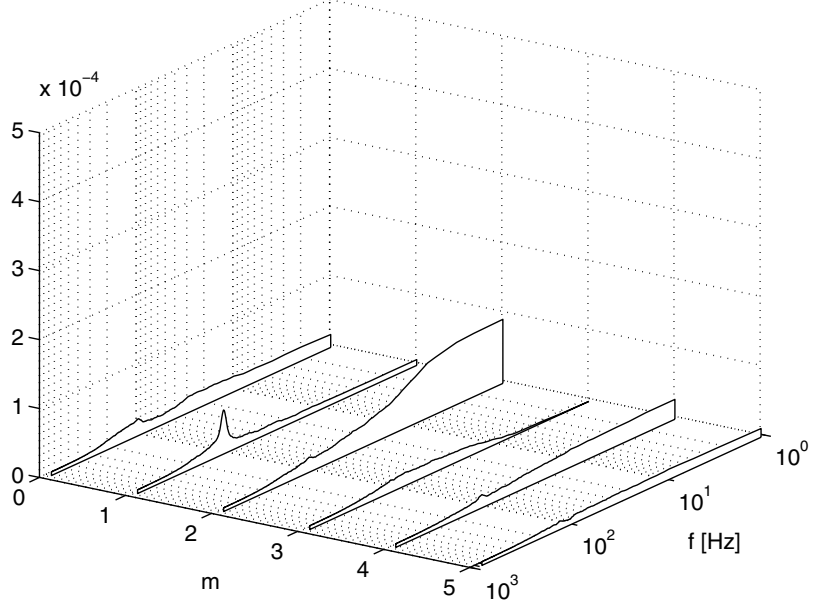

d)

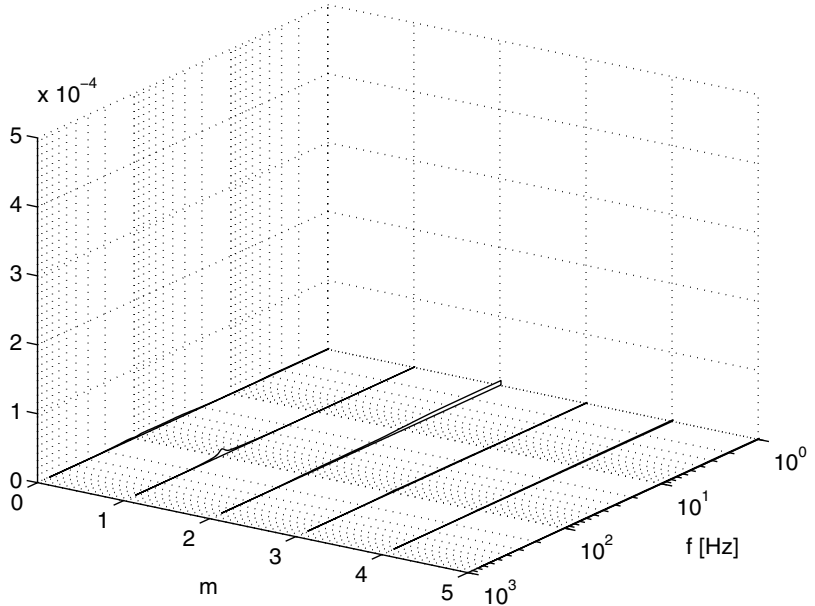

f) 
$S_{x, x}\left(r, r^{\prime} ; m ; f\right)+S_{r, r}\left(r, r^{\prime} ; m ; f\right)+S_{\theta, \theta}\left(r, r^{\prime} ; m ; f\right)$. As shown in Fig. 5, azimuthal mode 2 has more turbulence kinetic energy than the other azimuthal modes. The peak of azimuthal mode 2 is at near-zero frequency and reaches its largest value at $r=3 \Delta r$. The strong azimuthal mode 1 peak at the shedding frequency is also present. A very weak contribution from the vortex shedding also exists in azimuthal modes 0 and 2 at the shedding frequency. The general features are very similar to the distribution of the streamwise fluctuations, which is due to the relatively large contribution of the latter to the total energy.

\section{B. Modally Decomposed Two-Point Cross-Correlations}

The modally decomposed two-point cross-correlations for the first four azimuthal Fourier modes are shown in Fig. 6 in the same manner previously suggested by Wänström [33]. (Note that absolute value of the modally decomposed two-point correlations are only plotted in the figure.) These results represent the full Reynolds stress tensor decomposed in the azimuthal direction using Fourier series expansion. These were obtained by integrating the two-point crossspectra $S_{i, j}\left(r, r^{\prime} ; m ; f\right)$ over the frequency. $S_{r, \theta}\left(r, r^{\prime} ; m ; f\right)$ and $S_{\theta, r}\left(r, r^{\prime} ; m ; f\right)$ were computed using Eqs. (22) and (23), respectively, and then integrated over the frequency in the same manner.

The modally decomposed turbulence kinetic energy is the diagonal of the figures from the left bottom corner, showing the kinetic energy due to the streamwise fluctuations, to the right top corner, presenting the contribution of the azimuthal fluctuations. The contribution of the azimuthal fluctuations to the turbulence kinetic energy is smaller than the kinetic energy caused by the other two
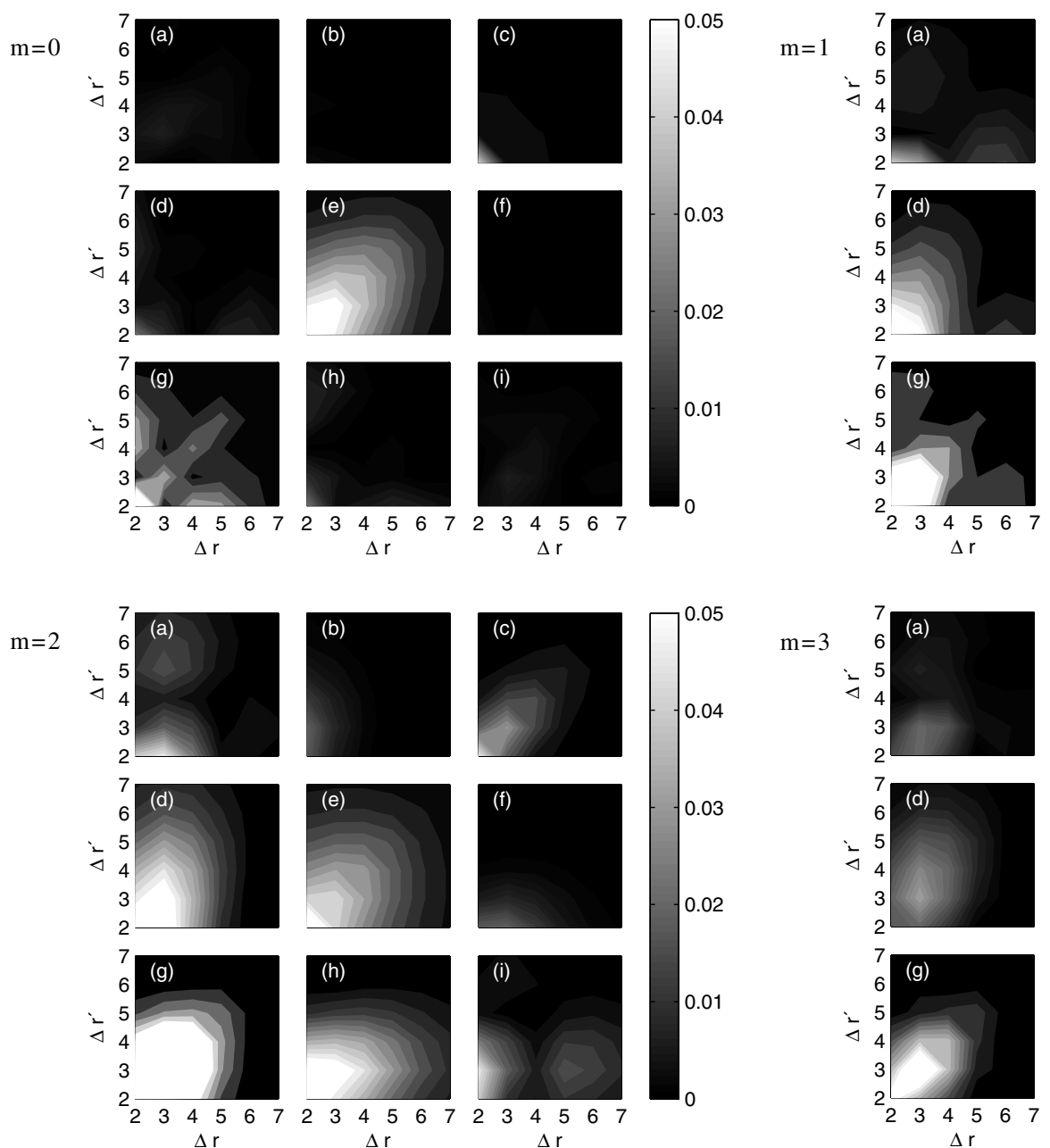
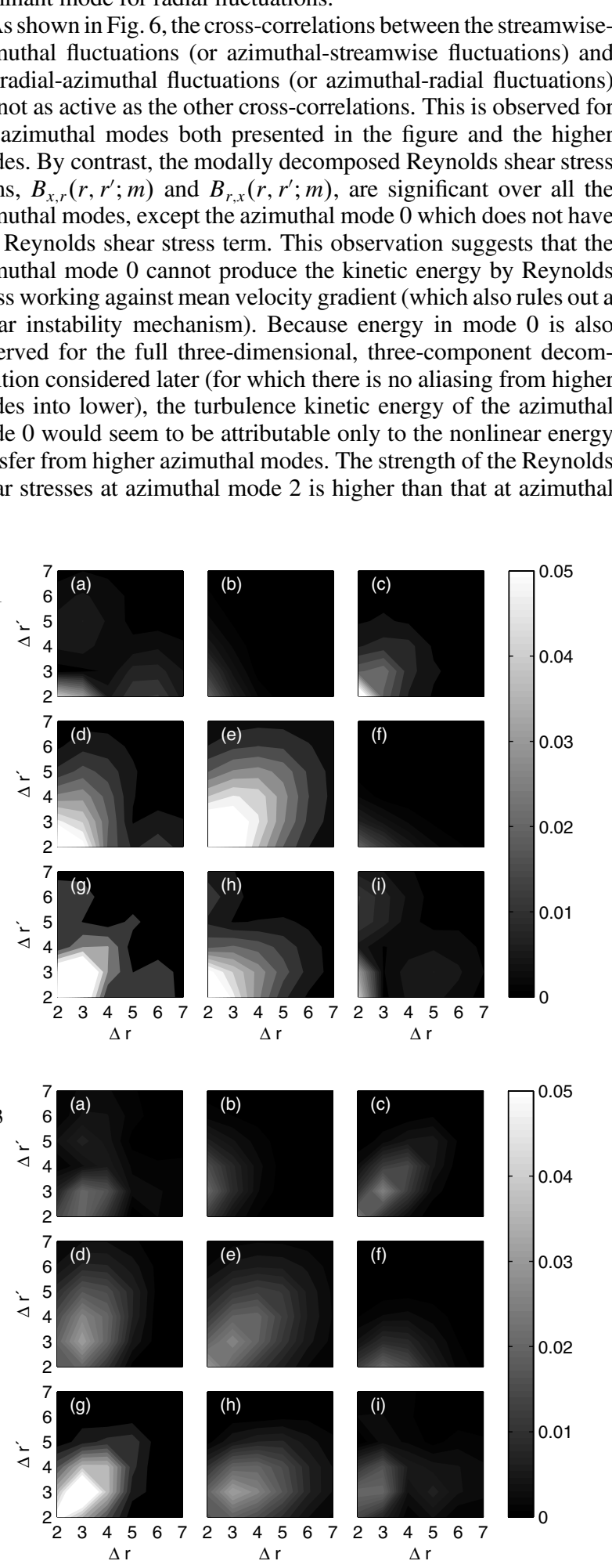

Fig. 6 Magnitudes of the modally decomposed two-point cross-correlations for the azimuthal Fourier modes $m=0,1,2$, and 3: a) $B_{\theta, x}\left(r, r^{\prime} ; m\right)$, b) $B_{\theta, r}\left(r, r^{\prime} ; m\right)$, c) $B_{\theta, \theta}\left(r, r^{\prime} ; m\right)$, d) $B_{r, x}\left(r, r^{\prime} ; m\right)$, e) $B_{r, r}\left(r, r^{\prime} ; m\right)$, f) $B_{r, \theta}\left(r, r^{\prime} ; m\right)$, g) $B_{x, x}\left(r, r^{\prime} ; m\right)$, h) $B_{x, r}\left(r, r^{\prime} ; m\right)$, i) $B_{x, \theta}\left(r, r^{\prime} ; m\right)$. 
mode 1 , and decreases for the higher azimuthal modes. This is consistent with the fact that the single point correlations involving the azimuthal velocity are identically zero due to the axisymmetry, whereas it is the correlation between the streamwise and radial velocity that is the primary source of energy production from the mean flow gradient.

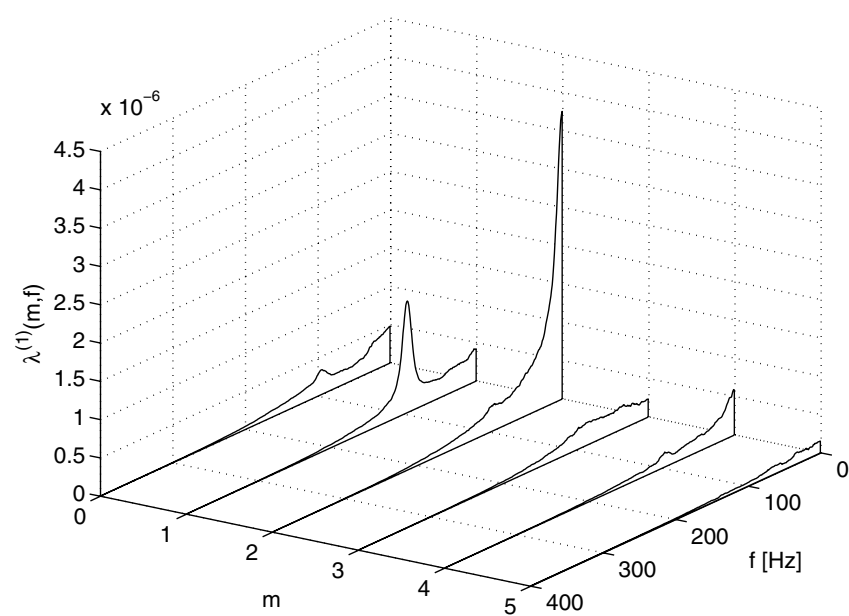

a) Eigenspectra of the first pod mode of $\mathrm{POD}_{u}$

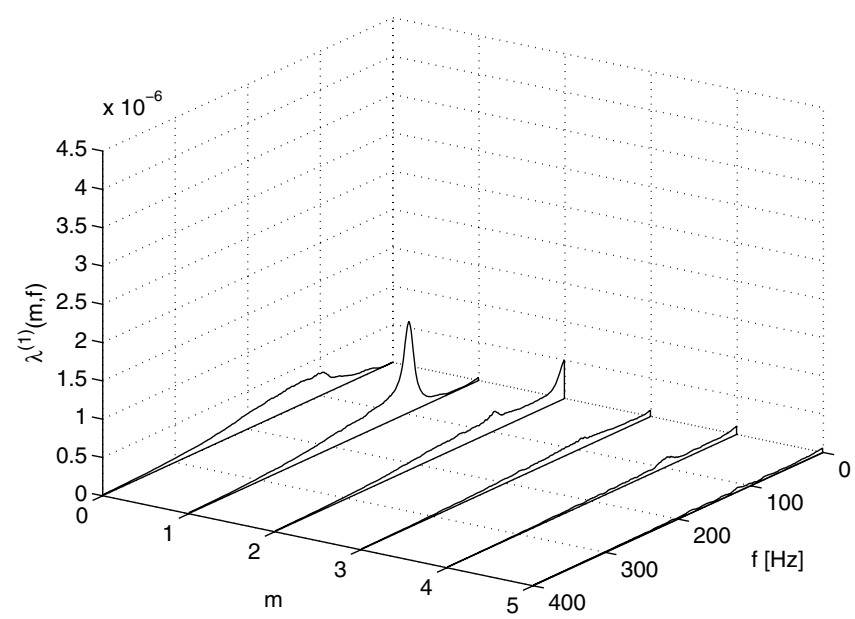

c) Eigenspectra of the first pod mode $\mathrm{POD}_{v}$

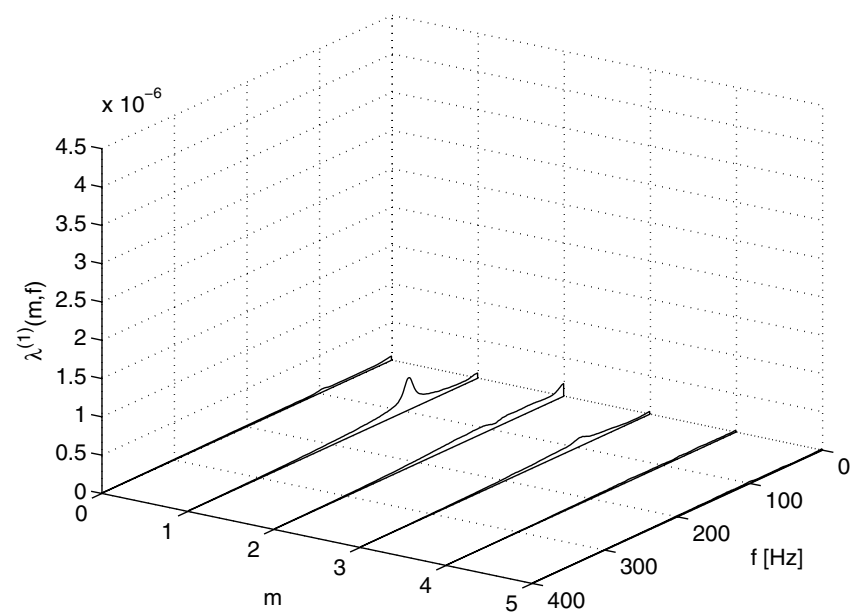

e) Eigenspectra of the first pod mode of $\mathrm{POD}_{w}$

\section{One-Component Scalar Proper Orthogonal Decomposition}

First, the eigenspectrum $\lambda^{(1)}(m, f)$ of the one-component scalar proper orthogonal decomposition of the streamwise fluctuations as a function of azimuthal mode number $(m)$ and frequency $(f)$ is presented in Fig. 7a. Only the first six azimuthal modes and first $400 \mathrm{~Hz}$ of the spectrum are shown in the figure, because eigenvalues

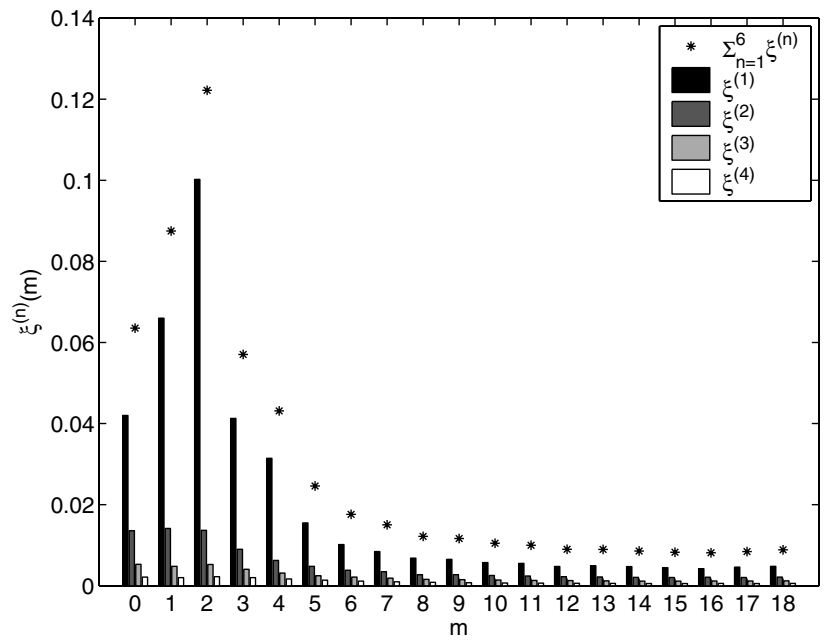

b) Normalized eigenvalue distribution of $\mathrm{POD}_{u}$

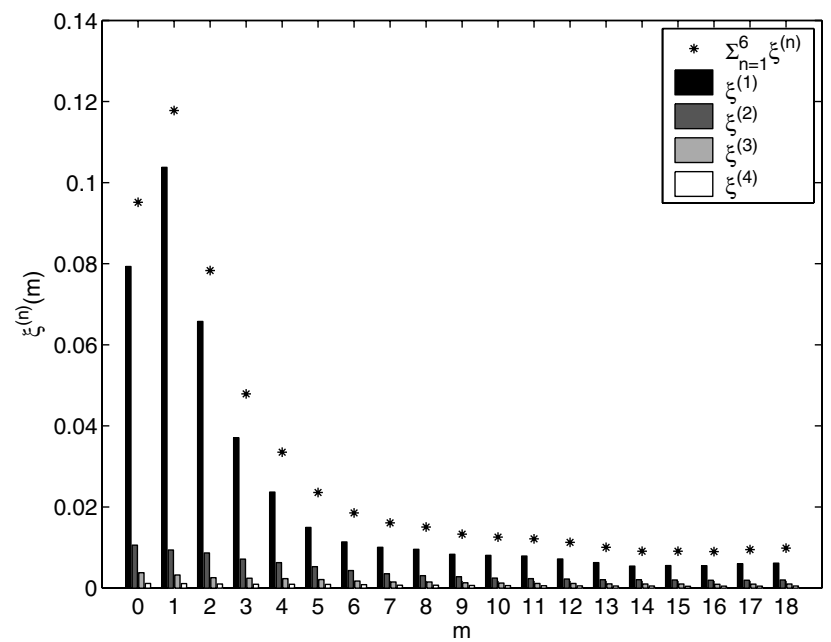

d) Normalized eigenvalue distribution of $\mathrm{POD}_{v}$

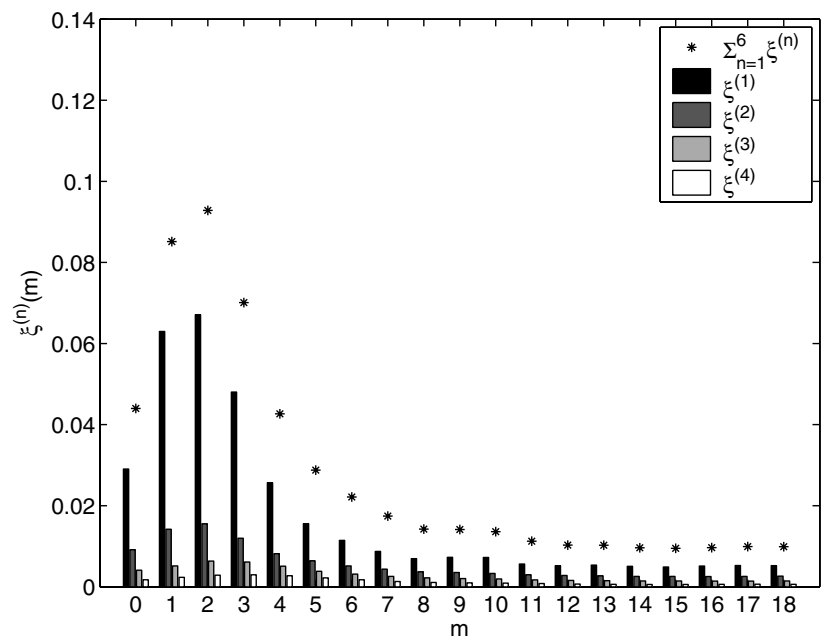

f) Normalized eigenvalue distribution of $\mathrm{POD}_{w}$

Fig. 7 One-component scalar proper orthogonal decomposition. 
at higher azimuthal modes and frequencies are not as important as the first ones, in terms of the magnitude. There are two distinct peaks in the eigenspectrum: the first peak appears in azimuthal mode 1 and is at the frequency corresponding to the shedding frequency. The peak at near-zero frequency is in the azimuthal mode 2 . The previous analysis of the eigenspectra for different downstream positions done by Johansson and George [16] is in excellent agreement for the same downstream location at $x / \bar{D}=50$.

The eigenspectra for all POD modes have been integrated over frequency $f$ to show the distribution of kinetic energy over the azimuthal modes. These are presented in Fig. $7 \mathrm{~b}$ in their normalized form. Normalization of eigenvalues is done using the following equation:

$$
\xi^{(n)}(m)=\frac{\int_{0}^{\infty} \lambda^{(n)}(m, f) \mathrm{d} f}{\sum_{m=0}^{M-1} \sum_{n}^{N} \int_{0}^{\infty} \lambda^{(n)}(m, f) \mathrm{d} f}
$$

where $M$ and $N$ are the maximum number of the azimuthal modes and POD modes, respectively, and $f$ stands for frequency. Each bar represents the turbulence energy of the POD modes at the corresponding azimuthal Fourier mode. For the clarity in the figure, first four POD modes for each azimuthal Fourier mode are presented. The $*$ sign shows the total kinetic energy of the azimuthal modes, which are computed by summing all POD modes for each azimuthal mode numbers. As also shown by Johansson and George [16], azimuthal mode 2 dominates the other azimuthal modes in terms of total energy contribution to the turbulent kinetic energy. Notice that for the one-component scalar decomposition, the total energy in the system is only due to that one-component of the fluctuations, and so summation over all $m$ is unity.

Following in the same manner, the first eigenspectrum $\lambda^{(1)}(m, f)$ of the one-component scalar decomposition of the radial component of the fluctuations is given in Fig. 7c. The function of both azimuthal modes and the frequency is given by $\lambda^{(1)}(m, f)$. There is only one distinct peak which appears at the shedding frequency, and it is an azimuthal mode 1 peak. Other than this phenomenon, there is no significant contribution to the kinetic energy budget from the radial fluctuations. It could be concluded that the radial fluctuations are linked to the vortex shedding at the disk. Another interesting result Fig. 7c reveals is the very low, or almost zero, magnitude of azimuthal mode 1 at near-zero frequencies. Azimuthal mode 1 is almost shutoff until the effect of the shedding begins to affect the distributions.

The eigenspectra integrated over frequency can be seen in Fig. 7d. This is also a normalized version of the integrated eigenspectra using Eq. (24), but this time based on the radial fluctuations only. As seen in Fig. $\overline{7 c}$, azimuthal mode 1 has the largest amount of energy for the first $\overline{\mathrm{POD}}$ mode. Therefore, it is not surprising to see the integrated eigenspectrum peaking at azimuthal mode 1 .

The first eigenspectrum of the one-component scalar decomposition of the azimuthal fluctuations, for the first six azimuthal Fourier modes and up to $400 \mathrm{~Hz}$ as before, are plotted in Fig. 7e. As seen in this figure, there is just one azimuthal mode 1 peak at the shedding frequency and it is very small when compared with the POD of the streamwise and radial fluctuations.

Figure $7 \mathrm{f}$ shows the integrated eigenspectra over the frequency $f$ as a result of $\mathrm{POD}_{w}$. The eigenspectra are also normalized by Eq. (24). As can be seen in the figure, the total contribution of azimuthal mode 2 is slightly larger than the total contribution of azimuthal mode 1 .

\section{Two-Component Vectorial Proper Orthogonal Decomposition}

Figures $8 \mathrm{a}, \underline{8 \mathrm{c}}$, and $\underline{8 \mathrm{e}}$, show the eigenspectra of twocomponent vectorial decomposition of the streamwise-radial, streamwise-azimuthal, and radial-azimuthal fluctuations, respectively. The three-dimensional eigenspectra in these figures show that the distribution is similar to the distribution of the one-component scalar decomposition of streamwise fluctuations. The azimuthal fluctuations do not have any significant effect on the distribution of eigenvalues. This is simply related to the contribution of the individual fluctuating components to the turbulent kinetic energy of the flow. As the eigenspectrum of the first POD mode of $\mathrm{POD}_{u}$ shows, it is the largest in magnitude, in contrast with that of $\mathrm{POD}_{w}$, which is the smallest in magnitude. Therefore, 1) when streamwise fluctuations are included in the analysis, the result is similar to the one from streamwise fluctuations alone, and 2) when azimuthal fluctuations are included in the analysis, the result is similar to the result obtained by the other pairs' analysis alone.

Figures $\underline{8 b}, \underline{8 d}$, and $\underline{8 f}$ present the normalized eigenspectra integrated over frequency $\bar{f}$ for the combination listed in the previous paragraph. Here, the denominator contains only the energy associated with the two components of the fluctuations. It can be seen that for the two-component vectorial decomposition of the streamwise and radial fluctuations, and for that of the streamwise and azimuthal fluctuations, the maximum is at azimuthal mode 2, but for that of the radial and azimuthal fluctuations, the peak has shifted to azimuthal mode 1 . The relative contribution of azimuthal mode 2 of the integrated eigenspectra distribution of the streamwise and radial fluctuations is smaller than for the streamwise fluctuations alone because azimuthal mode 1 is the dominant mode for the decomposition of the radial fluctuations. Therefore, more energy will be carried by azimuthal mode 1 compared with the single streamwise component case. The same features are observed on the other pairs too.

\section{E. Three-Component Vectorial Proper Orthogonal Decomposition}

The eigenspectra of the first POD mode of the three-component vectorial decomposition, as functions of azimuthal mode number and frequency, are plotted in Fig. 9a. As it can be seen in the figure, there is almost no difference between the results of the one-component scalar decompositions of the streamwise fluctuations, Fig. 7a, and the three-component vectorial decomposition of all components of the fluctuations. There are two distinct peaks in the spectrum: one is an azimuthal mode 1 peak at the shedding frequency, and the other one is an azimuthal mode 2 peak at the near-zero frequency. These findings are in agreement with the previous findings of Johansson and George [16]. On the other hand, the difference between the peaks of azimuthal modes 1 and 2 is smaller when all three components of the fluctuations are included. This is because the components added to system with dominant modes, other than the dominant mode of the streamwise fluctuations, rearrange the energy distribution over the modes. Unlike the far jet results, however, the effect on the far wake does not affect which mode corresponds to the peak.

Figure 10a shows the comparison between the eigenspectra of the three-component vectorial decomposition and the summation of the eigenspectra of one-component scalar decompositions of the streamwise, radial, and azimuthal fluctuations. Figure $10 \mathrm{~b}$ shows the two-component vectorial decomposition, including streamwise and radial fluctuations, and summation of the eigenspectra of onecomponent scalar decomposition of the streamwise and radial fluctuations. Figure $10 \mathrm{c}$ shows the two-component vectorial decomposition, including streamwise and azimuthal decompositions, and summation of the eigenspectra of one-component scalar decomposition of the streamwise and azimuthal fluctuations. Figure 10d shows the two-component vectorial decomposition consisting of radial and azimuthal components of velocity fluctuations, and summation of corresponding one-component scalar decompositions of radial and azimuthal components of the fluctuating velocity. These eigenspectra are obtained by summing the eigenspectra $\lambda^{(n)}(m, f)$ over the POD modes $n$ and azimuthal mode numbers $m$. There is remarkable agreement between the two-component vectorial decomposition and the summation of the one-component scalar decomposition of corresponding velocity components forming the vectorial decomposition. The eigenvalues of the three-component vectorial decomposition are found to be slightly higher than the summation of all eigenvalues from the onecomponent scalar decompositions of the streamwise, radial, and azimuthal fluctuations. Somewhat similar comparisons, for only two-component vectorial and one-component scalar decompositions, were previously documented by Delville [ $\underline{8}]$ for the turbulent 
plane mixing layer. Even though first, second, and third POD modes of one-component scalar decompositions of different velocity components for the plane mixing layer were quite different, he also reported an agreement between two-component vectorial decomposition and summation of the corresponding one-component scalar decompositions. He further noted that eigenspectra of the first POD

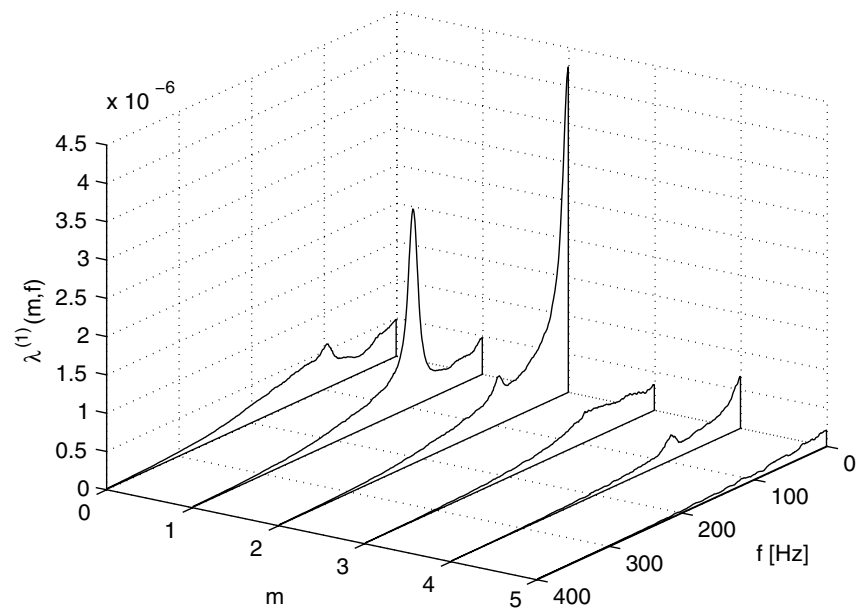

a) Eigenspectra of the first pod mode of $\mathrm{POD}_{u v}$

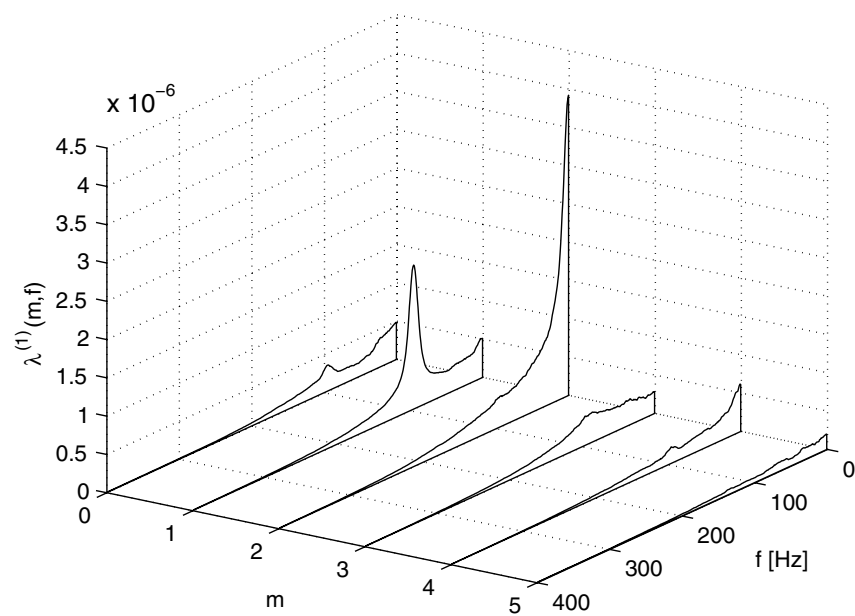

c) Eigenspectra of the first pod mode of $\mathrm{POD}_{u w}$

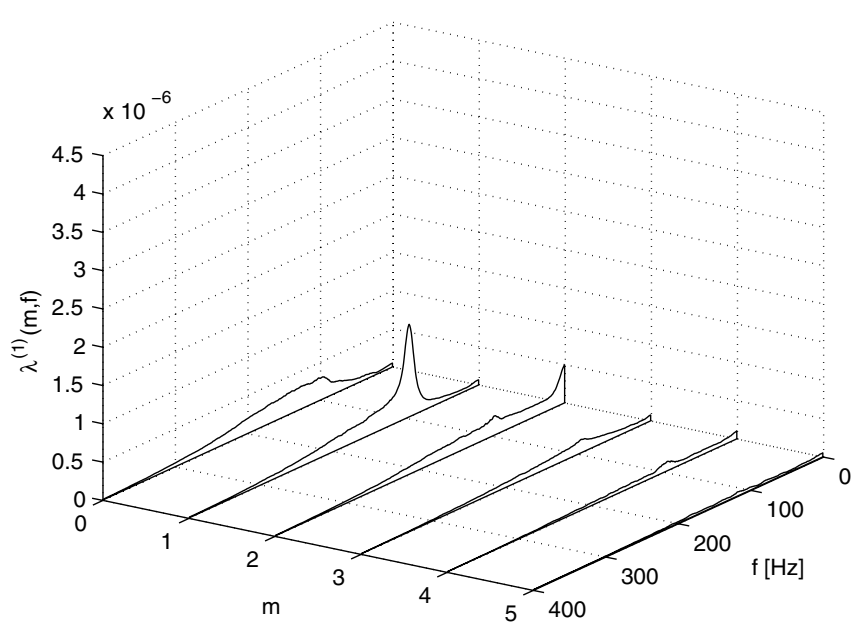

e) Eigenspectra of the first pod mode of $\mathrm{POD}_{v w}$ mode of the two-component vectorial POD is greater than or equal to the summation of the eigenspectra of the one-component scalar POD of the fluctuations included in the kernel of two-component vectorial POD. Our findings presented in Fig. 10 also show that eigenspectra of the vectorial decompositions are almost equal or slightly larger than those of the summation of the individual one-component scalar

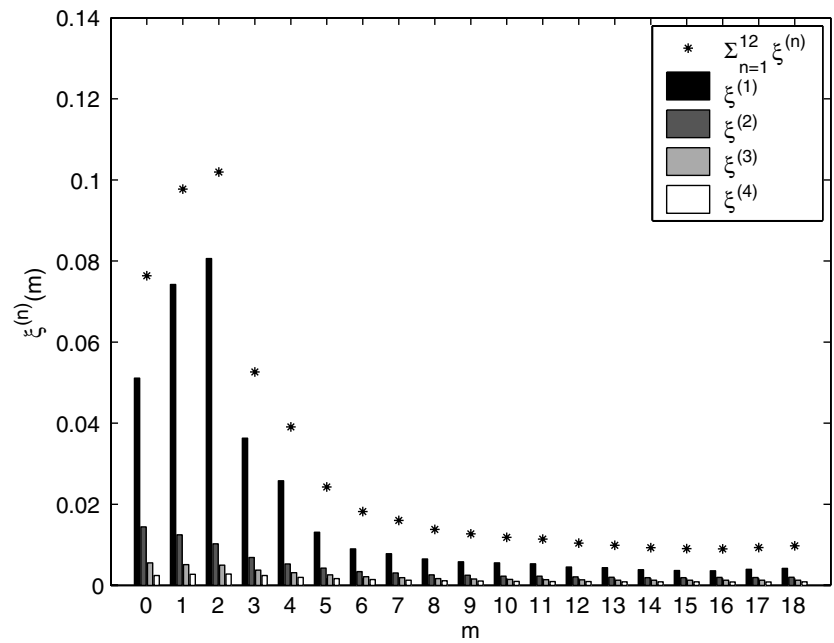

b) Normalized eigenvalue distribution of $\mathrm{POD}_{u v}$

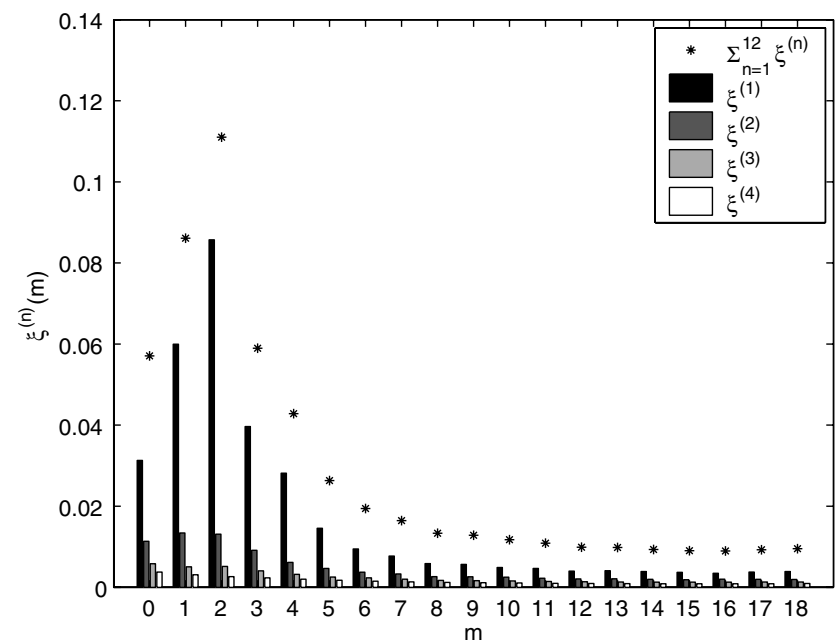

d) Normalized eigenvalue distribution of POD $_{u w}$

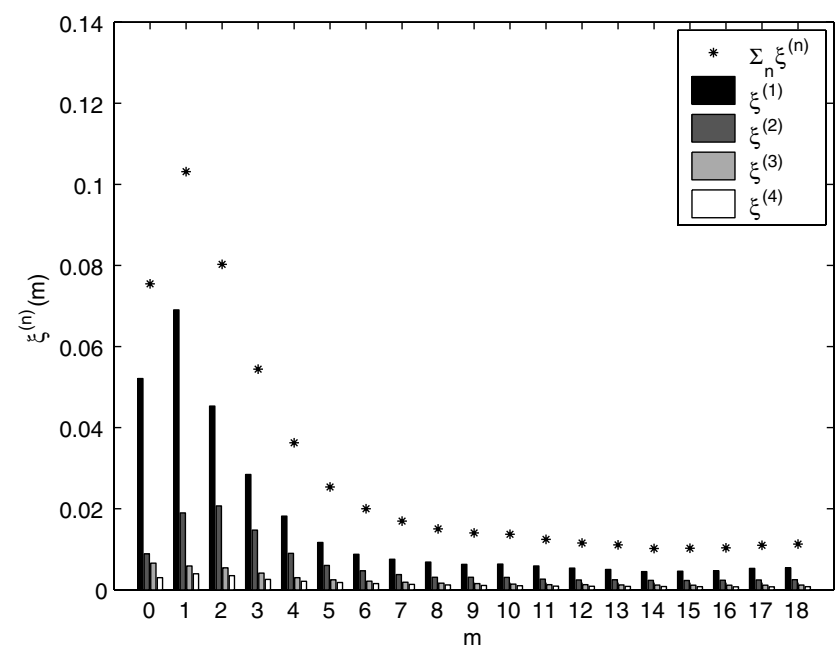

f) Normalized eigenvalue distribution of $\mathrm{POD}_{v w}$

Fig. 8 Two-component vectorial proper orthogonal decomposition. 


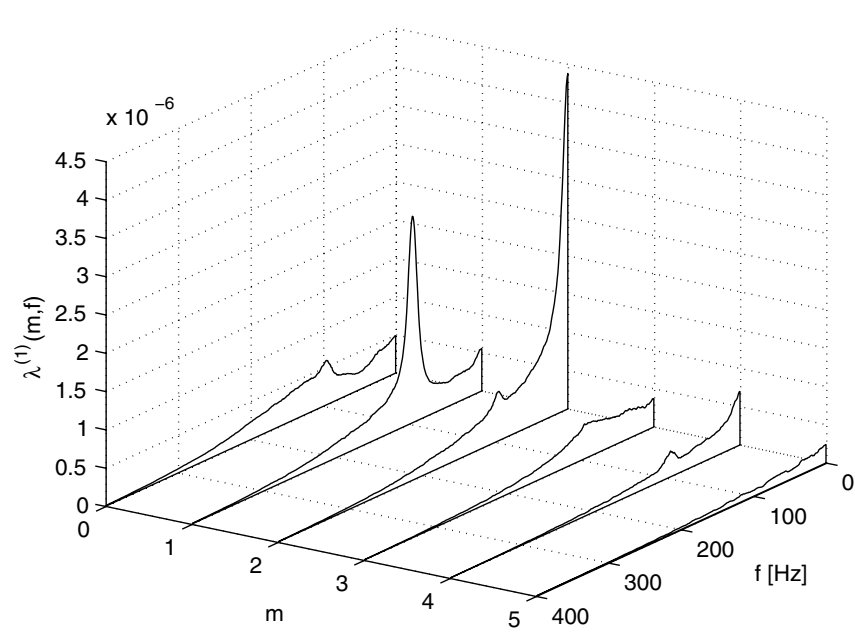

a) Eigenspectrum of the first pod mode of POD $_{u v w}$

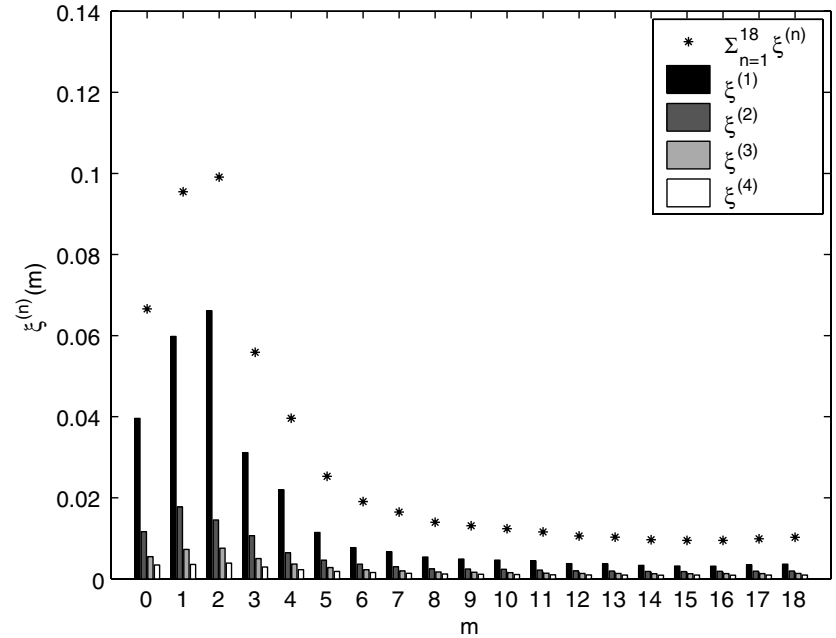

b) Normalized eigenvalue distribution of POD $_{u v w}$

Fig. 9 Three-component vectorial proper orthogonal decomposition.

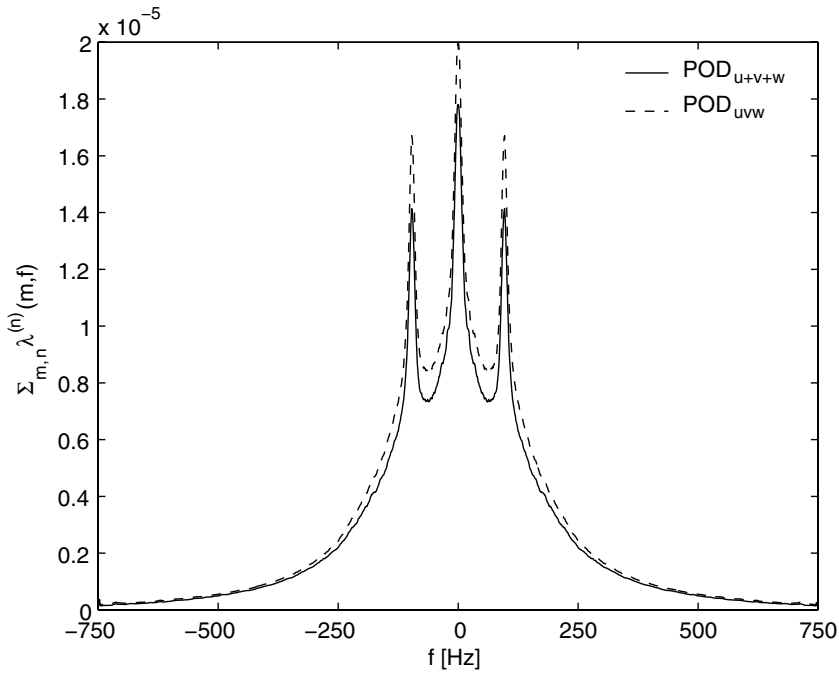

a)

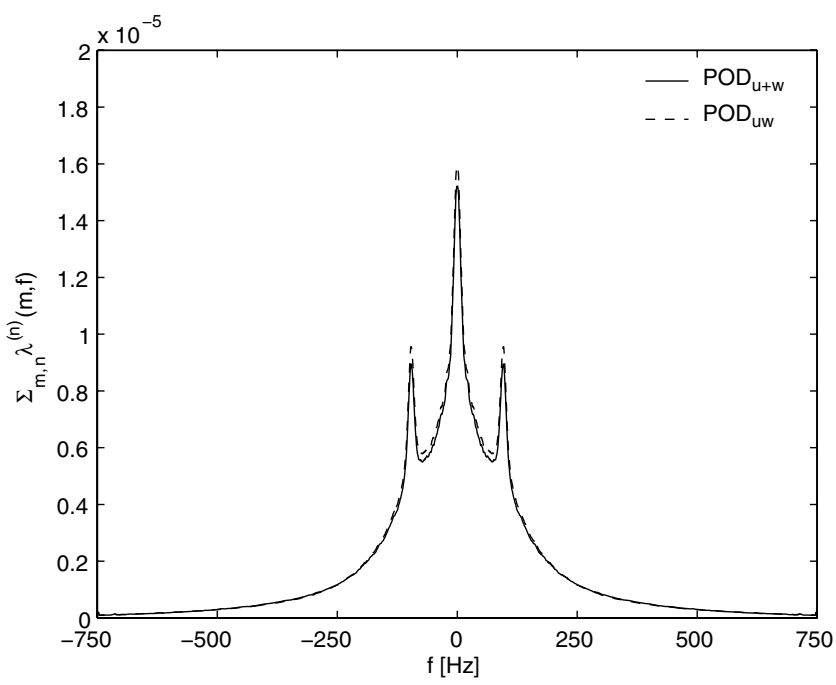

c)

Fig. 10 Integrated eigenspectrum over azimuthal and POD modes as function of frequency $f$. Comparison between POD results of different kernel construction.

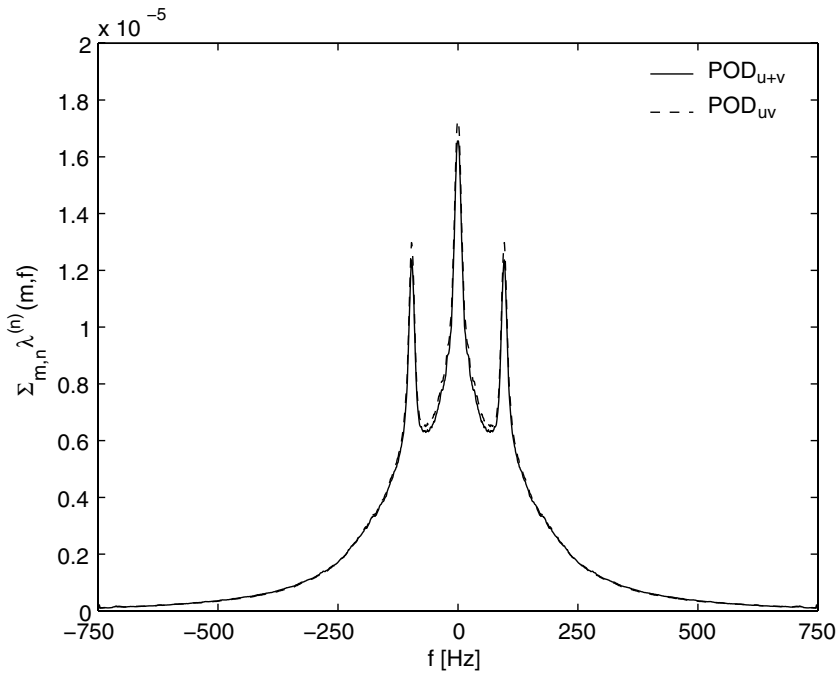

b)

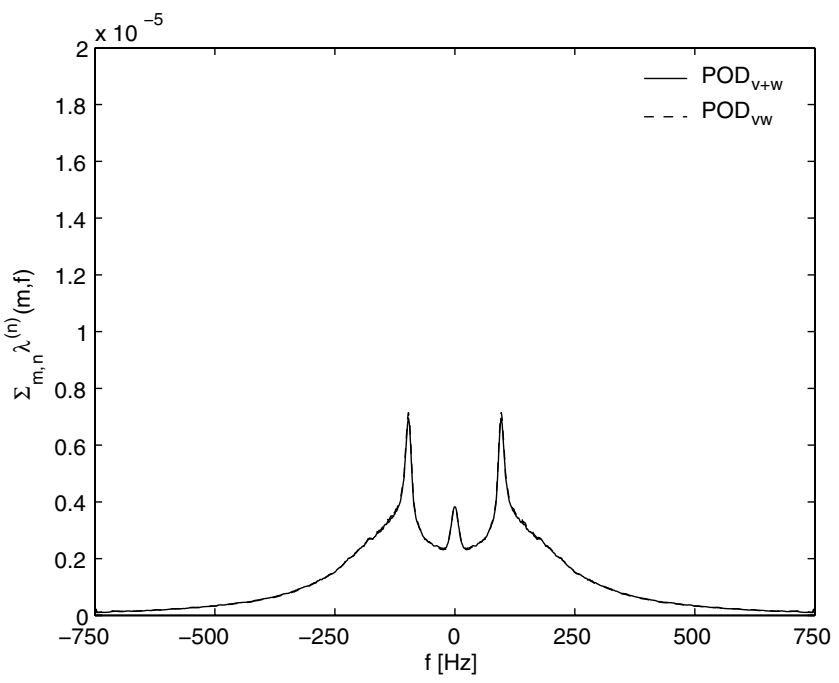

d) 
decompositions of the fluctuating velocities included in the vectorial decomposition.

\section{Conclusions}

In this paper, the one-component scalar proper orthogonal decomposition, the two-component vectorial proper orthogonal decomposition, and the three-component vectorial proper orthogonal decomposition were studied for the axisymmetric turbulent wake. First, the modally decomposed two-point crossspectra, together with the modally decomposed two-point crosscorrelations, were investigated to see how the turbulence kinetic energy is distributed over different frequencies and azimuthal Fourier modes. The results show that azimuthal mode 2 is the largest in the resulting POD eigenvalue distribution. In addition to this, the two-point cross-spectra indicated an azimuthal mode 1 peak at the shedding frequency and an azimuthal mode 2 peak at near-zero frequency. These results were consistent with the POD analysis of the axisymmetric turbulent wake of Johansson and George [16] based on only streamwise velocity measurements.

The one-component scalar POD were also performed for all fluctuating velocity components. The data and analysis for the streamwise component were in agreement with the previous studies. The one-component scalar decompositions of the radial and azimuthal fluctuations were performed to examine how their contributions differed from that of the streamwise component, and how their contributions differed from the full vector POD. Most interesting was that the radial scalar POD peaked at azimuthal mode 1 .

Different combinations of the two-component vectorial POD were also studied. The most obvious result for the two-component analysis was that whenever the streamwise fluctuations were included in the kernel of the integral equation, then the results were essentially the same as the one-component scalar decomposition of the streamwise fluctuations. The second finding was that the azimuthal fluctuations have only a very weak effect on the results when the two-component vectorial POD is performed.

Finally, the three-component vectorial POD was performed on the data using all nine components of the two-point velocity crossspectral tensor. The result was similar to that of the one-component scalar decomposition. The only difference was the distribution of energy over the azimuthal modes in terms of percentages. Azimuthal mode 2 dominance was more pronounced when only the streamwise component was used, because the effective energy content of the flow increased by adding more components into the computation.

In summary, in axisymmetric wake turbulence, the one-, two-, or three-component proper orthogonal decompositions give similar results in terms of the azimuthal modal distribution of the eigenvalues, or turbulent kinetic energy. The azimuthal mode 2 is always dominant when the streamwise fluctuations are included in the analysis. The similarity in the eigenvalue distribution of one- and three-component POD for the wake can be attributed to how turbulence is produced in this flow. As documented in Johansson et al. [21], the transport equations for the Reynolds stresses show that there is only one turbulence kinetic energy production term in the axisymmetric far wake, and it is in the normal stress term due to streamwise fluctuations $\left\langle u^{2}\right\rangle$. Therefore, as long as the streamwise velocity is involved in computation, no significant difference is expected in these one-, two-, and three-component implementations. This is quite contrary to what has been observed in the axisymmetric jet by Iqbal and Thomas [17] and Wänström et al. [20], where different modal peaks were observed depending on which decomposition was used, or even which partial integral or sum was performed. Clearly, in spite of the axisymmetry of both flows, they are very different structurally and dynamically.

\section{Acknowledgments}

The authors would like to thank Maja Wänström of Chalmers University of Technology, B. Anders Pettersson Reif of Norwegian Defence Research Establishment (FFI), Jonathan Naughton of
University of Wyoming, and Jöel Delville of Laboratoire d'Etudes Aérodynamiques for useful discussions. The reviewers are also thanked for their constructive comments. This work was supported in part by the Swedish Research Council (Vetenskapsrådet), grant number 2001-2641, and in part by the Swedish Energy Agency (Energimyndigheten), grant number P21715-1. M. Tutkun acknowledges the partial support from the Center of Excellence grant from the Norwegian Research Council to the Center for Biomedical Computing.

\section{References}

[1] Lumley, J. L., "Structure of Inhomogeneous Turbulent Flows," Atmospheric Turbulence and Radio Wave Propagation, edited by A. M. Yaglom, and V. I. Tatarsky, Publishing House Nauka, Moscow, USSR, 1967, pp. 166-176.

[2] George, W. K., "Insight into the Dynamics of Coherent Structures from a Proper Orthogonal Decomposition," Structure of Near Wall Turbulence, Proceedings of Symposium on Near Wall Turbulence, edited by S. Kline, Hemisphere, New York, 1988, pp. 168-180.

[3] Holmes, P., Lumley, J. H., and Berkooz, G., Turbulence, Coherent Structures, Dynamical Systems and Symmetry, Cambridge Univ. Press, Cambridge, England, U.K., 1996.

[4] Glauser, M. N., "Coherent Structures in the Axisymmetric Turbulent Jet Mixing Layer," Ph.D. Dissertation, State Univ. of New York at Buffalo, Buffalo, NY, 1987.

[5] Herzog, S., "Large Scale Structure in the Near-Wall Region of Turbulent Pipe Flow," Ph.D. Dissertation, Cornell Univ., Ithaca, NY, 1986.

[6] Glauser, M. N., and George, W. K., "Orthogonal Decomposition of the Axisymmetric Jet Mixing Layer Including Azimuthal Dependence," Advances in Turbulence, edited by G. Comte-Bellot, and J. Mathieu, Springer, Berlin, 1987, pp. 357-366.

[7] Glauser, M. N., Leib, S. J., and George, W. K., "Coherent Structures in the Axisymmetric Turbulent Jet Mixing Layer," Turbulent Shear Flows 5, edited by F. Durst, B. E. Launder, J. H. Lumley, F. W. Schmidt, and J. H. Whitelaw, Springer-Verlag, Berlin, 1985, pp. 134-145.

[8] Delville, J., "Characterization of the Organization in Shear Layers via the Proper Orhogonal Decomposition," Applied Scientific Research, Vol. 53, Nos. 3-41994, pp. 263-281.

doi:10.1007/BF00849104

[9] Delville, J., Ukeiley, L., Cordier, L., Bonnet, J. P., and Glauser, M., "Examination of Large-Scale Structures in a Turbulent Plane Mixing Layer, Part 1: Proper Orthogonal Decomposition," Journal of Fluid Mechanics, Vol. 391, July 1999, pp. 91-122. doi:10.1017/S0022112099005200

[10] Gordeyev, S. V., and Thomas, F. O., "Coherent Structure in the Turbulent Planar Jet, Part 1: Extraction of Proper Orthogonal Decomposition Eigenmodes and Their Self-Similarity," Journal of Fluid Mechanics, Vol. 414, July 2000, pp. 145-194. doi:10.1017/S002211200000848X

[11] Gordeyev, S. V., and Thomas, F. O., "Coherent Structure in the Turbulent Planar Jet, Part 2: Structural Topology via POD Eigenmode Projection," Journal of Fluid Mechanics, Vol. 460, June 2002, pp. 349380 .

doi:10.1017/S0022112002008364

[12] Citriniti, J. H., and George, W. K., "Reconstruction of the Global Velocity Field in the Axisymmetric Mixing Layer Utilizing the Proper Orthogonal Decomposition," Journal of Fluid Mechanics, Vol. 418, Sept. 2000, pp. 137-166. doi: $10.1017 /$ S0022112000001087

[13] Johansson, P. B. V., George, W. K., and Woodward, S. H., "Proper Orthogonal Decomposition of an Axisymmetric Turbulent Wake Behind a Disk," Physics of Fluids, Vol. 14, No. 7, 2002, pp. 2508-2514. doi:10.1063/1.1476301

[14] Jung, D., Gamard, S., and George, W. K., "Downstream Evolution of the Most Energetic Modes in a Turbulent Axisymmetric Jet at High Reynolds Number, Part 1: The Near-Field Region," Journal of Fluid Mechanics, Vol. 514, Sept. 2004, pp. 173-204. doi:10.1017/S0022112004000163

[15] Gamard, S., Jung, D., and George, W. K., "Downstream Evolution of the Most Energetic Modes in a Turbulent Axisymmetric Jet at High Reynolds Number, Part 2: The Far-Field Region," Journal of Fluid Mechanics, Vol. 514, Sept. 2004, pp. 205-230. doi:10.1017/S0022112004000175

[16] Johansson, P. B. V., and George, W. K., "Far Downstream Evolution of the High Reynolds Number Axisymmetric Wake Behind a Disk, Part 2: Slice Proper Orthogonal Decomposition," Journal of Fluid Mechanics, 
Vol. 555, May 2006, pp. 387-408. doi: $10.1017 / \mathrm{S} 0022112006009517$

[17] Iqbal, M. O., and Thomas, F. O., "Coherent Structure in a Turbulent Jet via a Vector Implementation of the Proper Orthogonal Decomposition," Journal of Fluid Mechanics, Vol. 571, Jan. 2007, pp. 281-326. doi:10.1017/S0022112006003351

[18] Glauser, M. N., and George, W. K., "Orthogonal Decomposition of the Axisymmetric Jet Mixing Layer Utilizing Cross-Wire Velocity Measurements," Proceedings of the Sixth Symposium on Turbulent Shear Flows, Springer-Verlag, Toulouse, France, 1987, pp. 10.1.110.1.6.

[19] Citriniti, J. H., Experimental Investigation into the Dynamics of the Axisymmetric Mixing Layer Utilizing the Proper Orthogonal Decomposition, Ph.D. Dissertation, State Univ. of New York at Buffalo, Buffalo, NY, 1996.

[20] Wänström, M., George, W. K., and Meyer, K.-E., "Stereoscopic PIV and POD Applied to Far Turbulent Axisymmetric Jet," AIAA Paper 2006-3368, 2006.

[21] Johansson, P. B. V., George, W. K., and Gourlay, M. J., "Equilibrium Similarity, Effects of Initial Conditions and Local Reynolds Number on the Axisymmetric Wake," Physics of Fluids, Vol. 15, No. 3, 2003, pp. 603-617. doi:10.1063/1.1536976

[22] Johansson, P. B. V., and George, W. K., "Far Downstream Evolution of the High Reynolds Number Axisymmetric Wake Behind a Disk, Part 1: Single Point Statistics," Journal of Fluid Mechanics, Vol. 555, May 2006, pp. 363-385 doi: $10.1017 / \mathrm{S} 0022112006009529$

[23] Glauser, M. N., and George, W. K., "Application of Multipoint Measurements for Flow Characterization," Experimental Thermal and Fluid Science, Vol. 5, No. 5, 1992, pp. 617-632. doi:10.1016/0894-1777(92)90018-Z

[24] Citriniti, J. H., and George, W. K., "Reduction of Spatial Aliasing by Long Hot-Wire Anemometer Probes," Experiments in Fluids, Vol. 23, No. 3, 1997, pp. 217-224. doi:10.1007/s003480050105

[25] Tan-atichat, J., Woodward, S. H., and George, W. K., "Use of Computers for Data Acquisition and Processing," Handbook of Fluids and Fluids Engineering, edited by J. A. Schetz, and A. E. Fuhs, Wiley, New York, 1996.

[26] Lumley, J. L., Stochastic Tools in Turbulence, Academic Press, New York, 1970

[27] Tutu, N. K., and Chevray, R., "Cross-Wire Anemometry in High Intensity Turbulence," Journal of Fluid Mechanics, Vol. 71, No. 4, 1975 , pp. $785-800$. doi:10.1017/S0022112075002856

[28] Shabbir, A., Beuther, P. D., and George, W. K., "X-Wire Response in Turbulent Flows of High-Intensity Turbulence and Low Mean Velocities," Experimental Thermal and Fluid Science, Vol. 12, No. 1, 1996, pp. 52-56. doi:10.1016/0894-1777(95)00068-2

[29] Champagne, F. H., Sleicher, C. A., and Wehrmann, O. H., "Turbulence Measurements with Inclined Hot-Wires, Part 1: Heat Transfer Experiments with Inclined Hot-Wire," Journal of Fluid Mechanics, Vol. 28, No. 1, 1967, pp. 153-175 doi:10.1017/S002211206700196X

[30] George, W. K., Beuther, P. D., and Shabbir, A., "Polynomial Calibrations for Hot Wires in Thermally Varying Flows," Experimental Thermal and Fluid Science, Vol. 2, No. 2, 1989, pp. 230-235. doi:10.1016/0894-1777(89)90038-1

[31] Lumley, J. L., "Interpretation of Time Spectra Measured in HighIntensity Shear Flows," Physics of Fluids, Vol. 8, No. 6, June 1965 pp. 1056-1062. doi:10.1063/1.1761355

[32] George, W. K., Hussein, H. J., and Woodward, S. H., "Evaluation of the Effect of Fluctuating Convection Velocity on the Validity of Taylor's Hypothesis," Proceedings of the 10th Australasian Fluid Mechanics Conference 5.2, edited by A. E. Perry, Univ. of Melbourne, Melbourne, Australia, Dec. 1989, pp. 11.5-11.8.

[33] Wänström, M., "Using Stereoscopic PIV to Investigate the Crossplane Modal Composition of a Far Turbulent Axisymmetric Jet," Licentiate Thesis, Chalmers Univ. of Technology, Göteborg, Sweden, 2006.

R. Rangel Associate Editor 